\title{
ARITHMETICAL RANK OF THE CYCLIC AND BICYCLIC GRAPHS
}

\author{
MARGHERITA BARILE, DARIUSH KIANI, FATEMEH MOHAMMADI, \\ AND SIAMAK YASSEMI
}

\begin{abstract}
We show that for the edge ideals of the graphs consisting of one cycle or two cycles of any length connected through a vertex or a path, the arithmetical rank equals the projective dimension.
\end{abstract}

\section{INTRODUCTION}

For any homogeneous ideal $I$ of a polynomial ring $R=K\left[x_{1}, \ldots, x_{n}\right]$ there exists a graded minimal finite free resolution

$$
0 \rightarrow \bigoplus_{j} R(-j)^{\beta_{p j}} \rightarrow \ldots \rightarrow \bigoplus_{j} R(-j)^{\beta_{1 j}} \rightarrow R \rightarrow R / I \rightarrow 0
$$

of $R / I$, in which $R(-j)$ denotes the graded free module obtained by shifting the degrees of elements in $R$ by $j$. The numbers $\beta_{i j}$, which we shall refer to as the $i$ th Betti numbers of degree $j$ of $R / I$, are independent of the choice of the graded minimal finite free resolution. We also define the $i$ th Betti number of $I$ as $\beta_{i}:=$ $\sum \beta_{i j}$.

Given a polynomial ring $R$ over a field, and a graph $G$ having the set of indeterminates as its vertex set $V(G)$ and the set of edges $E(G)$, one can associate with $G$ a monomial ideal of $R$ : this ideal is generated by the products of the vertices of each edge in $E(G)$, and is hence generated by squarefree quadratic monomials. It is called the edge ideal $I(G)$ of $G$, and has been intensively studied by Simis, Vasconcelos and Villarreal in [17. The arithmetical rank (ara), i.e., the least number of elements of $R$ which generate a given monomial ideal up to radical, is in general bounded below by its projective dimension $(p d)$, i.e., by the length of every minimal free resolution of the quotient of $R$ with respect to the ideal. The simplicial complex $\Delta_{G}$ of a graph $G$ is defined by

$$
\Delta_{G}=\{A \subseteq V(G) \mid A \text { is an independent set in } G\},
$$

where $A$ is an independent set in $G$ if none of its elements are adjacent. Note that $\Delta_{G}$ is precisely the Stanley-Reisner simplicial complex of $I(G)$.

For any simplicial complex $\Delta$ on the vertex set $V(\Delta)$, the Alexander dual of $\Delta$ is the simplicial complex defined by

$$
\Delta^{*}:=\{F \subseteq V(\Delta) \mid V(\Delta) \backslash F \notin \Delta\}
$$

2000 Mathematics Subject Classification. 13A15; 13F55; 13D02; 05C38.

Key words and phrases. Arithmetical rank, projective dimension, edge ideals, set-theoretic complete intersection ideals.

D. Kiani was supported in part by a grant from IPM (No. 87200116).

S. Yassemi was supported in part by a grant from IPM (No. 87130211). 
The link of a face $F \in \Delta$ is defined as the simplicial complex

$$
\operatorname{Link}_{\Delta} F:=\{G \in \Delta \mid G \cup F \in \Delta \text { and } G \cap F=\emptyset\} .
$$

In recent times, the projective dimension has been determined for large classes of edge ideals, where it is independent of the ground field: in Jacques' thesis it was computed for acyclic graphs (see also [12]), but also for the graphs $C_{n}$, consisting of one cycle of length $n$. Jacques, in [11, Theorem 6.1.8], using Hochster's formula [9, showed that for a graph $G$, the Betti numbers are

$$
\beta_{i, d}(G)=\sum_{H \subset G,|V(H)|=d} \operatorname{dim}_{k} \tilde{H}_{i-2}(\varepsilon(H) ; K) .
$$

Then he used $(*)$ for providing formulas for the graded Betti numbers of special classes of graphs including lines, cycles and complete graphs. He proved the following theorems.

Theorem A [11, Lemma 8.2.7] The reduced homology of the disjoint union of the cyclic graph $C_{n}$ and any non empty graph $G$ may be expressed as follows:

$$
\tilde{H}_{i}\left(\varepsilon\left(C_{n} \cup G\right) ; K\right)= \begin{cases}\tilde{H}_{i-\frac{2 n+1}{3}}(\varepsilon(G) ; K), & \text { if } n \equiv 1 \bmod 3 \\ \tilde{H}_{i-\frac{2 n-1}{3}}(\varepsilon(G) ; K), & \text { if } n \equiv 2 \bmod 3 .\end{cases}
$$

Theorem B [11, Corollary 7.6.30] The non zero Betti numbers in degree $n$ and the projective dimension of $C_{n}$ in degree $n$ are the following:

$\beta_{\frac{2 n}{3}, n}=2, \quad$ and $p d I\left(C_{n}\right)=\frac{2 n}{3}$, if $n \equiv 0 \bmod 3$,

$\beta_{\frac{2 n+1}{3}, n}=1$, and $p d I\left(C_{n}\right)=\frac{2 n+1}{3}$, if $n \equiv 1 \bmod 3$,

$\beta_{\frac{2 n-1}{3}, n}=1$, and $p d I\left(C_{n}\right)=\frac{2 n-1}{3}$, if $n \equiv 2 \bmod 3$.

Theorem C [11, Lemma 8.1.3] The reduced homology of the disjoint union of the line graph $L_{n}$ and any non empty graph $G$ may be expressed as follows:

$$
\tilde{H}_{i}\left(\varepsilon\left(L_{n} \cup G\right) ; K\right)=\left\{\begin{array}{cl}
\tilde{H}_{i-\frac{2 n}{3}}(\varepsilon(G) ; K), & \text { if } n \equiv 0 \bmod 3 \\
0, & \text { if } n \equiv 1 \bmod 3 \\
\tilde{H}_{i-\frac{2 n-1}{3}}(\varepsilon(G) ; K), & \text { if } n \equiv 2 \bmod 3 .
\end{array}\right.
$$

From the proof of [11, Corollary 7.7.35] one can derive the following result.

Theorem D [11, Corollary 7.7.35] The projective dimension of the line graph is independent of the characteristic of the chosen field and is

$$
\text { pd } I\left(L_{n}\right)=\left\{\begin{array}{cl}
\frac{2 n}{3} & \text { if } n \equiv 0 \bmod 3 \\
\frac{2 n-2}{3} & \text { if } n \equiv 1 \bmod 3 \\
\frac{2 n-1}{3} & \text { if } n \equiv 2 \bmod 3
\end{array}\right.
$$

All Betti numbers of $L_{n}$ in degree $n$ are zero if $n \equiv 1 \bmod 3$. Otherwise the non zero Betti numbers of degree $n$ of $L_{n}$ are

$$
\begin{gathered}
\beta_{\frac{2 n}{3}, n} I\left(L_{n}\right)=1, \text { if } n \equiv 0 \bmod 3, \\
\beta_{\frac{2 n-1}{3}, n} I\left(L_{n}\right)=1 \text {, if } n \equiv 2 \bmod 3 .
\end{gathered}
$$

In [6] an explicit formula is given for the Betti numbers of a special kind of bipartite graphs, the so-called Ferrers graphs. In [2] it is shown that the arithmetical rank equals the projective dimension for a special class of acyclic graphs, in 3 , that 
this is also true for all Ferrers graphs. In the present paper we prove that the same equality holds for all cyclic and bicyclic graphs. By bicyclic graph we mean a graph which consists of two cycles that have exactly one vertex in common or are connected by a path. In particular, we will see that the projective dimension of the edge ideals of these graphs does not depend on the characteristic of the ground field.

\section{THE ARITHMETICAL RANK OF CYCLIC GRAPHS}

Let $K$ be a field, and consider the polynomial $\operatorname{ring} R=K\left[x_{1}, \ldots, x_{n}\right]$, where $n \geq 3$. Let $C_{n}$ be the graph on the vertex set $\left\{x_{1}, \ldots, x_{n}\right\}$ whose set of edges is $\left\{\left\{x_{1}, x_{2}\right\},\left\{x_{2}, x_{3}\right\}, \ldots,\left\{x_{n-1}, x_{n}\right\},\left\{x_{1}, x_{n}\right\}\right\}$. Then its edge ideal is the following ideal of $R$ :

$$
I\left(C_{n}\right)=\left(x_{1} x_{2}, x_{2} x_{3}, \ldots, x_{n-1} x_{n}, x_{1} x_{n}\right) .
$$

We will show that for all $n, p d I\left(C_{n}\right)=\operatorname{ara} I\left(C_{n}\right)$. In general, for any ideal $I$ of $R$ we have that $c d I \leq \operatorname{ara} I$, where $c d$ denotes the local cohomological dimension (see 8], Example 2, p. 414) and, whenever $I$ is a monomial ideal, $p d I=c d I$ (see [14, Theorem 1). Hence it will suffice to show that, for all $n$, ara $I\left(C_{n}\right) \leq p d I\left(C_{n}\right)$, i.e., to produce $p d I\left(C_{n}\right)$ elements of $R$ generating $I\left(C_{n}\right)$, up to radical. Among the available tools, we have, on the one hand, Jacques' result providing explicit formulas for the projective dimension of $I\left(C_{n}\right)$.

On the other hand, we know that a finite set of elements of $R$ which generate a given ideal up to radical can be constructed according to the following criterion, which is due to Schmitt and Vogel.

Lemma 2.1. ([16], p. 249) Let $P$ be a finite subset of elements of $R$. Let $P_{0}, \ldots, P_{r}$ be subsets of $P$ such that

(i) $\bigcup_{i=0}^{r} P_{i}=P$;

(ii) $P_{0}$ has exactly one element;

(iii) if $p$ and $p^{\prime}$ are different elements of $P_{i}(0<i \leq r)$ there is an integer $i^{\prime}$ with $0 \leq i^{\prime}<i$ and an element in $P_{i^{\prime}}$ which divides $p p^{\prime}$.

We set $q_{i}=\sum_{p \in P_{i}} p^{e(p)}$, where $e(p) \geq 1$ are arbitrary integers. We will write $(P)$ for the ideal of $R$ generated by the elements of $P$. Then we get

$$
\sqrt{(P)}=\sqrt{\left(q_{0}, \ldots, q_{r}\right)}
$$

We have to distinguish between three cases, depending on the residue of $n$ modulo 3. The cases $n \equiv 0,1 \bmod 3$ can be settled by a direct application of Lemma 2.1] The case $n \equiv 2 \bmod 3$ is more interesting, since it needs some additional non trivial computations on the generators.

Proposition 2.2. Suppose that $n=3 m$, for some integer $m$. Set $q_{0}=x_{1} x_{2}$, $q_{1}=x_{1} x_{3 m}+x_{2} x_{3}$, and, for $1 \leq i \leq m-1$, set

$$
\begin{aligned}
q_{2 i} & =x_{3 i+1} x_{3 i+2} \\
q_{2 i+1} & =x_{3 i} x_{3 i+1}+x_{3 i+2} x_{3 i+3} .
\end{aligned}
$$

Then

$$
I\left(C_{n}\right)=\sqrt{\left(q_{0}, \ldots, q_{2 m-1}\right)} .
$$

In particular, ara $I\left(C_{n}\right) \leq 2 m$. 
Proof. For all $i=0, \ldots, m-1$, the monomial $q_{2 i}$ divides the product of the two summands of $q_{2 i+1}$. By Lemma 2.1 it follows that

$$
\left(x_{3 i} x_{3 i+1}, x_{3 i+1} x_{3 i+2}, x_{3 i+2} x_{3 i+3}\right)=\sqrt{\left(q_{2 i}, q_{2 i+1}\right)} .
$$

This implies the claim.

Using the same arguments as in the proof of Proposition 2.2. from Lemma 2.1 we can deduce the next result.

Proposition 2.3. Suppose that $n=3 m+1$, for some integer $m$. Set $q_{0}=x_{1} x_{2}$, $q_{1}=x_{1} x_{3 m+1}+x_{2} x_{3}$, and, for $1 \leq i \leq m-1$, set

$$
\begin{aligned}
q_{2 i} & =x_{3 i+1} x_{3 i+2} \\
q_{2 i+1} & =x_{3 i} x_{3 i+1}+x_{3 i+2} x_{3 i+3},
\end{aligned}
$$

and, finally, $q_{2 m}=x_{3 m} x_{3 m+1}$. Then

$$
I\left(C_{n}\right)=\sqrt{\left(q_{0}, \ldots, q_{2 m}\right)} .
$$

In particular, ara $I\left(C_{n}\right) \leq 2 m+1$.

Proposition 2.4. Suppose that $n=3 m+2$, for some integer $m$. Set $q_{0}=x_{1} x_{2}$, $q_{1}=x_{2} x_{3}+x_{4} x_{5}$, and, for $1 \leq i \leq m-1$, set

$$
\begin{aligned}
q_{2 i} & =x_{3 i} x_{3 i+1}+x_{3 i+2} x_{3 i+3} \\
q_{2 i+1} & =x_{3 i+2} x_{3 i+3}+x_{3 i+4} x_{3 i+5},
\end{aligned}
$$

and, finally, $q_{2 m}=x_{1} x_{3 m+2}+x_{3 m} x_{3 m+1}$. Then

$$
I\left(C_{n}\right)=\sqrt{\left(q_{0}, \ldots, q_{2 m}\right)} .
$$

In particular, ara $I\left(C_{n}\right) \leq 2 m+1$.

Proof. The claim for $m=1$ was proven in [2, Example 1. So let $m \geq 2$. Set $J_{m}=\left(q_{0}, \ldots, q_{2 m}\right)$. It suffices to show that $I\left(C_{n}\right) \subset \sqrt{J_{m}}$. In this proof, for all $f, g \in R$, by abuse of notation we will write $f \equiv g$ whenever $f-g$ or $f+g$ belongs to $J_{m}$, and, $f \equiv_{q_{i}} g$ whenever $f-g$ or $f+g$ is divisible by $q_{i}$. In this way, $f \equiv g$ or $f \equiv_{q_{i}} g$ assures that $f \in J_{m}$ occurs if and only if $g \in J_{m}$. We first show that

$$
x_{1}^{2^{m}} x_{3 m+2}^{2^{m+1}} \in J_{m} .
$$

Set

$$
\begin{aligned}
u_{m} & =x_{1}^{2^{m-1}} x_{3 m+2}^{2^{m}}, \\
v_{m} & =x_{3} x_{4} x_{5} \prod_{i=2}^{m} x_{3 i}^{3 \cdot 2^{i-2}}, \\
w_{m} & =\left(x_{3 m} x_{3 m+1} x_{3 m+2}\right)^{2^{m-1}} .
\end{aligned}
$$

We prove that

$$
u_{m} \equiv v_{m} \equiv w_{m} .
$$

Note that $x_{1} x_{3 m+2} v_{m}$ is a multiple of $x_{1} x_{3 m+2} x_{4} x_{5}$, and

$$
x_{1} x_{3 m+2} x_{4} x_{5} \equiv_{q_{0}} x_{1} x_{3 m+2}\left(x_{2} x_{3}+x_{4} x_{5}\right) \in J_{m},
$$

whence we deduce that $x_{1} x_{3 m+2} v_{m} \in J_{m}$. Thus (2.2) will imply that

$$
x_{1}^{2^{m}} x_{3 m+2}^{2^{m+1}}=x_{1} x_{3 m+2} u_{m} \in J_{m},
$$


as claimed in (2.1). We prove (2.2) by induction on $m \geq 2$. First take $m=2$. We have $q_{2}=x_{3} x_{4}+x_{5} x_{6}, q_{3}=x_{5} x_{6}+x_{7} x_{8}$ and $q_{4}=x_{1} x_{8}+x_{6} x_{7}$, so that

$$
v_{2}=x_{3} x_{4} x_{5} x_{6}^{3} \equiv_{q_{2}} x_{5}^{2} x_{6}^{2} x_{6}^{2} \equiv_{q_{3}} x_{6}^{2} x_{7}^{2} x_{8}^{2}=w_{2} \equiv_{q_{4}} x_{1}^{2} x_{8}^{4}=u_{2},
$$

which shows (2.2) for $m=2$. Now suppose that $m>2$ and that the claim is true for $m-1$. We have:

$$
\begin{gathered}
v_{m}=v_{m-1} x_{3 m}^{3 \cdot 2^{m-2}} \equiv w_{m-1} x_{3 m}^{3 \cdot 2^{m-2}} \\
=\quad\left(x_{3 m-3} x_{3 m-2} x_{3 m-1}\right)^{2^{m-2}} x_{3 m}^{3 \cdot 2^{m-2}}=\left(x_{3 m-3} x_{3 m-2}\right)^{2^{m-2}} x_{3 m-1}^{2^{m-2}} x_{3 m}^{3 \cdot 2^{m-2}} \\
\equiv_{q_{2 m-2}} \quad\left(x_{3 m-1} x_{3 m}\right)^{2^{m-2}} x_{3 m-1}^{2^{m-2}} x_{3 m}^{3 \cdot 2^{m-2}}=\left(x_{3 m-1} x_{3 m}\right)^{2^{m-1}} x_{3 m}^{2 \cdot 2^{m-2}} \\
\equiv_{q_{2 m-1}} \quad\left(x_{3 m+1} x_{3 m+2}\right)^{2^{m-1}} x_{3 m}^{2^{m-1}}=\left(x_{3 m} x_{3 m+1}\right)^{2^{m-1}} x_{3 m+2}^{2^{m-1}}=w_{m} \\
\equiv_{q_{2 m}} \quad\left(x_{1} x_{3 m+2}\right)^{2^{m-1}} x_{3 m+2}^{2^{m-1}}=x_{1}^{2^{m-1}} x_{3 m+2}^{2^{m}}=u_{m} .
\end{gathered}
$$

This completes the proof of (2.2) and of (2.1). We have thus shown that

$$
x_{1} x_{3 m+2} \in \sqrt{J_{m}} .
$$

But then

$$
x_{3 m} x_{3 m+1}=q_{2 m}-x_{1} x_{3 m+2} \in \sqrt{J_{m}} .
$$

In general, whenever, for some $i \in\{2, \ldots, m\}$,

$$
x_{3 i} x_{3 i+1} \in \sqrt{J_{m}},
$$

from the fact that $x_{3 i} x_{3 i+1}$ divides $x_{3 i-1} x_{3 i} \cdot x_{3 i+1} x_{3 i+2}$, i.e., the product of the summands of $q_{2 i-1}$, by Lemma 2.1 one deduces that

$$
x_{3 i-1} x_{3 i} \in \sqrt{J_{m}} .
$$

Since $x_{3 i-3} x_{3 i-2}=q_{2 i-2}-x_{3 i-1} x_{3 i}$, this in turn implies that

$$
x_{3 i-3} x_{3 i-2} \in \sqrt{J_{m}} .
$$

Finally, since $x_{3 i-3} x_{3 i-2}$ divides $x_{3 i-4} x_{3 i-3} \cdot x_{3 i-2} x_{3 i-1}$, i.e., the product of the summands of $q_{2 i-3}$, by Lemma 2.1 we again conclude that

$$
x_{3 i-2} x_{3 i-1} \in \sqrt{J_{m}} .
$$

Therefore, since (2.5) implies (2.6), (2.7) and (2.8), for all $i=2, \ldots, m$, from (2.4) one can derive by descending induction on $h$, that $x_{h} x_{h+1} \in \sqrt{J_{m}}$ for all $h=3, \ldots, 3 m+1$. In particular we have that $x_{3} x_{4} \in \sqrt{J_{m}}$, which, together with $q_{1} \in J_{m}$, yields $x_{2} x_{3} \in \sqrt{J_{m}}$ by Lemma 2.1. This, together with (2.4) and $q_{0} \in J_{m}$, shows that $I\left(C_{n}\right) \subset \sqrt{J_{m}}$, as claimed.

Theorem B and Propositions 2.2, 2.3, 2.4 imply our main result. 
6MARGHERITA BARILE, DARIUSH KIANI, FATEMEH MOHAMMADI, AND SIAMAK YASSEMI

Theorem 2.5. Let $n \geq 3$ be an integer. Then

$$
\text { ara } I\left(C_{n}\right)=\operatorname{pd} I\left(C_{n}\right)=\left\{\begin{array}{ccc}
\frac{2 n}{3} & \text { if } & n \equiv 0 \bmod 3 \\
\frac{2 n+1}{3} & \text { if } & n \equiv 1 \bmod 3 \\
\frac{2 n-1}{3} & \text { if } & n \equiv 2 \bmod 3
\end{array}\right.
$$

Every ideal $I\left(C_{n}\right)$ is of pure height $\left\lceil\frac{n}{2}\right\rceil$, where $\lceil a\rceil$ denotes the least integer not less than $a$. Recall that an ideal is called a set-theoretic complete intersection if its arithmetical rank equals its height. In view of Theorem 2.5 we thus have the following.

Corollary 2.6. $I\left(C_{n}\right)$ is a set-theoretic complete intersection only for $n=3$ and $n=5$.

\section{THE ARITHMETICAL RANK OF BICYCLIC GRAPHS}

In this section by $\equiv$, we mean $\equiv(\bmod 3)$ and all equivalence relations will be considered modulo 3 . Let $a_{1}, \ldots, a_{s}$ be subsets of the finite set $V$. Define $\varepsilon\left(a_{1}, \ldots, a_{s} ; V\right)$ to be the simplicial complex which has vertex set $\bigcup_{i=1}^{s}\left(V \backslash a_{i}\right)$ and maximal faces $V \backslash a_{1}, \ldots, V \backslash a_{s}$. Let $\Delta=\Delta_{G}$, and let $F \in \Delta^{*}$ and $e_{1}, \ldots, e_{r}$ be all the edges of $G$ which are disjoint from $F$. Then $\operatorname{Link}_{\Delta^{*}} F=\varepsilon\left(e_{1}, \ldots, e_{r} ; V(G) \backslash F\right)$ by [12, Proposition 3.3]. According to [11, Proposition 6.1.6], associating $F$ with the induced subgraph $H$ of $G$ on the vertex set $V(G) \backslash F$ defines a bijection between the faces of $\Delta^{*}$ and the set of induced subgraphs of $G$ which have at least one edge. Let $H$ be an induced subgraph of the graph $G$. If $H$ is associated with the face $F$ of $\Delta^{*}$ as described above, we write $\varepsilon(H)$ for $\varepsilon\left(e_{1}, \ldots, e_{s} ; V\right)$, where $e_{1}, \ldots, e_{s}$ are the edges of $H$ and $V$ is the vertex set $V(G) \backslash F$ (or equivalently the vertex set of $H)$. In this section, using $(*)$, we find explicit descriptions of the projective dimension of all bicyclic graphs. For every vertex $u$ of a graph $G$ we denote by $N_{G}(u)$ the set of vertices adjacent to $u$. In the proof of our main results we will use the Mayer-Vietoris sequence for the reduced homology of simplicial complexes, which, for any pair $\Delta_{1}, \Delta_{2}$ of simplicial complexes, has the following form (see [11, Remark 6.2.13]):

$$
\begin{array}{r}
\ldots \rightarrow \tilde{H}_{i}\left(\Delta_{1} \cap \Delta_{2} ; K\right) \rightarrow \tilde{H}_{i}\left(\Delta_{1} ; K\right) \oplus \tilde{H}_{i}\left(\Delta_{2} ; K\right) \rightarrow \tilde{H}_{i}\left(\Delta_{1} \cup \Delta_{2} ; K\right) \rightarrow \\
\tilde{H}_{i-1}\left(\Delta_{1} \cap \Delta_{2} ; K\right) \rightarrow \ldots
\end{array}
$$

Lemma 3.1. For a graph $G$ with an edge $\{u, v\}$ such that $\operatorname{deg}(v)=1$, we have $\tilde{H}_{i}(\varepsilon(G) ; K)=0$, if some vertex in $N_{G}(u)$ has an adjacent vertex of degree one in $G$. Otherwise, $\tilde{H}_{i}(\varepsilon(G) ; K)=\tilde{H}_{i-t}(\varepsilon(H) ; K)$, where $t=\left|N_{G}(u)\right|$ and $H$ is the induced subgraph on $V(G) \backslash\left(\{u\} \cup N_{G}(u)\right)$, provided $H$ is non empty.

Proof. In this and in the following proofs we will omit the coefficient field in the homology groups. We set $V=V(G)$. Let $N_{G}(u)=\left\{v, u_{1}, \ldots, u_{t-1}\right\}$ and $\{u, v\},\left\{u, u_{1}\right\}, \ldots,\left\{u, u_{t-1}\right\}, e_{1}, \ldots, e_{r}$ be the edges of $G$. We can write $\varepsilon(G)=$ $E_{1} \cup E_{2}$, where $E_{1}=\varepsilon\left(\left\{u, u_{1}\right\}, \ldots,\left\{u, u_{t-1}\right\}, e_{1}, \ldots, e_{r} ; V\right)$ and $E_{2}=\varepsilon(\{u, v\} ; V)$. The intersection of these simplicial complexes is:

$E_{1} \cap E_{2}=\varepsilon\left(\left\{u, v, u_{1}\right\}, \ldots,\left\{u, v, u_{t-1}\right\},\{u, v\} \cup e_{1}, \ldots,\{u, v\} \cup e_{r} ; V\right)$ $=\varepsilon\left(\left\{u_{1}\right\}, \ldots,\left\{u_{t-1}\right\}, e_{1}, \ldots, e_{r} ; V \backslash(\{u, v\})\right)$ (see [12, Lemma 3.4]).

If there exists a vertex $v_{i}$ of degree one such that $\left\{u_{i}, v_{i}\right\} \in E(G)$, then without loss 
of generality we can assume that $e_{1}=\left\{u_{i}, v_{i}\right\}$. Then $E_{1} \cap E_{2}=\varepsilon\left(\left\{u_{1}\right\}, \ldots,\left\{u_{t-1}\right\}\right.$, $\left.\left\{u_{i}, v_{i}\right\}, e_{2}, \ldots, e_{r} ; V \backslash(\{u, v\})\right)=\varepsilon\left(\left\{u_{1}\right\}, \ldots,\left\{u_{t-1}\right\}, e_{2}, \ldots, e_{r} ; V \backslash(\{u, v\})\right)$, whose reduced homology is identically zero, since $v_{i} \in V \backslash(\{u, v\})$ and $v_{i}$ belongs to all faces of $E_{1} \cap E_{2}$. Otherwise, by [12, Lemma 3.5] we have $\tilde{H}_{i}\left(E_{1} \cap E_{2}\right)=$ $\tilde{H}_{i-t+1}(\varepsilon(H))$, for all $i$, where $H$ is the induced subgraph on $V \backslash\left(\{u\} \cup N_{G}(u)\right)$. Since $E_{2}$ is a simplex, $\tilde{H}_{i}\left(E_{2}\right)=0$ for all $i$. Also, $\tilde{H}_{i}\left(E_{1}\right)=0$ for all $i$, since $v$ belongs to all faces of $E_{1}$. Using the Mayer-Vietoris sequence (for $\Delta_{i}=E_{i}$ ) we deduce that $\tilde{H}_{i}(\varepsilon(G))=\tilde{H}_{i-1}\left(E_{1} \cap E_{2}\right)$, which completes the proof.

The next result can be deduced from Lemma 3.1 by a trivial inductive argument.

Corollary 3.2. Let $n \equiv 0$. Suppose that $L_{n}$ intersects graph $G$ only at one of its endpoints. Then, for all $i$, we have $\tilde{H}_{i}\left(\varepsilon\left(G \cup L_{n}\right)\right)=\tilde{H}_{i-\frac{2 n}{3}}\left(\varepsilon\left(G \backslash L_{n}\right)\right)$.

Theorem 3.3. Let $G$ be the graph which is a joint of two cycles $C_{n}$ and $C_{m}$ in a common vertex. Then the following hold:

(a) If $|V(G)| \equiv 1$, then $p d I(G)=$ ara $I(G)=\frac{2|V(G)|+1}{3}$.

(b) If $|V(G)| \equiv 0$, then pd $I(G)=\operatorname{ara} I(G)=\frac{2|V(\stackrel{3}{G})|}{3}$.

(c) If $|V(G)| \equiv 2$, then $p d I(G)=$ ara $I(G)=\frac{2|V(G)|+2}{3}$, for $m \equiv 0$, whereas pd $I(G)=\operatorname{ara} I(G)=\frac{2|V(G)|-1}{3}$ otherwise.

Proof. We will prove the claim by showing that the desired number is, on the one hand, a lower bound for $p d I(G)$, on the other hand, an upper bound for ara $I(G)$.

Let $V=V(G)$. Consider the labeling for $V$ such that $V\left(C_{n}\right)=\left\{y_{1}, y_{2}, \ldots, y_{n}\right\}$, and $V\left(C_{m}\right)=\left\{x_{1}, x_{2}, \ldots, x_{m}\right\}$, where $x_{1}=y_{1}$. Up to exchanging $m$ and $n$ we have the following cases.

Case $1 .|V| \equiv 0$ or 1 .

First let $n=3$. Then $m \equiv 1$ or $m \equiv 2$. In view of $(*)$ the $i$ th Betti number of degree $|V|$ is $\beta_{i,|V|}(G)=\operatorname{dim}_{k} \tilde{H}_{i-2}(\varepsilon(G) ; K)$. So we compute the reduced homology of $G$ of degree $|V|$. We can write $\varepsilon(G)=\varepsilon\left(\left\{x_{1}, x_{2}\right\}, \ldots,\left\{x_{m}, x_{1}\right\},\left\{x_{1}, y_{2}\right\},\left\{y_{2}, y_{3}\right\},\left\{y_{3}, x_{1}\right\}\right.$; $V)=E_{1} \cup E_{2}$, where $E_{1}=\varepsilon\left(\left\{x_{1}, x_{2}\right\}, \ldots,\left\{x_{m}, x_{1}\right\},\left\{x_{1}, y_{2}\right\},\left\{y_{3}, x_{1}\right\} ; V\right)$ and $E_{2}=\varepsilon\left(\left\{y_{2}, y_{3}\right\} ; V\right)$.

By [12, Lemma 3.4], the intersection of these simplicial complexes is:

$$
\begin{aligned}
E_{1} \cap E_{2} & =\varepsilon\left(\left\{x_{1}, x_{2}, y_{2}, y_{3}\right\}, \ldots,\left\{x_{1}, x_{m}, y_{2}, y_{3}\right\},\left\{x_{1}, y_{2}, y_{3}\right\} ; V\right) \\
& =\varepsilon\left(\left\{x_{1}\right\},\left\{x_{2}, x_{3}\right\}, \ldots,\left\{x_{m-1}, x_{m}\right\} ; V \backslash\left\{y_{2}, y_{3}\right\}\right) .
\end{aligned}
$$

By [12, Lemma 3.5], $\tilde{H}_{i}\left(E_{1} \cap E_{2}\right)=\tilde{H}_{i-1}\left(\varepsilon\left(L_{m-1}\right)\right)$, for all $i$. Since $E_{2}$ is a simplex, $\tilde{H}_{i}\left(E_{2}\right)=0$ for all $i$. Applying Lemma 3.1 to $E_{1}$ for $v=y_{2}$ (and $u=x_{1}$, so that $\left.N(u)=\left\{x_{2}, x_{m}, y_{2}, y_{3}\right\}\right)$, we obtain $\tilde{H}_{i}\left(E_{1}\right)=\tilde{H}_{i-4}\left(\varepsilon\left(L_{m-3}\right)\right)$, for all $i$.

If $m \equiv 1$, then $\tilde{H}_{i}\left(E_{1}\right)=0$ for all $i$ by Theorem $\mathrm{D}$ and $\left(^{*}\right)$ (since $m-3 \equiv 1$ ). By the Mayer-Vietoris sequence we deduce that, for all $i, \tilde{H}_{i}(\varepsilon(G))=\tilde{H}_{i-1}\left(E_{1} \cap E_{2}\right)$. Theorem $\mathrm{D}$ and $\left(^{*}\right)$ then show that $\tilde{H}_{i-2}\left(\varepsilon\left(L_{m-1}\right)\right) \neq 0$ (i.e., $\left.\tilde{H}_{i}(\varepsilon(G)) \neq 0\right)$ if and only if $i-2+2=\frac{2(m-1)}{3}$, if and only if $i=\frac{2|V|}{3}-2$. In view of $(*)$ we deduce that pd $I(G) \geq \frac{2|V|}{3}$.

If $m \equiv 2$, then $\tilde{H}_{i}\left(E_{1} \cap E_{2}\right)=0$ for all $i$ by Theorem $\mathrm{D}$ and $(*)($ since $m-1 \equiv 1)$. By the Mayer-Vietoris sequence we deduce that $\tilde{H}_{i}(\varepsilon(G))=\tilde{H}_{i}\left(E_{1}\right)$ for all $i$. Theorem $\mathrm{D}$ and $\left(^{*}\right)$ show that $\tilde{H}_{i}(\varepsilon(G)) \neq 0$ if and only if $i=\frac{2|V|+1}{3}-2$. In view of $\left(^{*}\right)$ we 
deduce that $p d I(G) \geq \frac{2|V|+1}{3}$.

So we can assume that $n \geq 4$. Moreover, since $n$ and $m$ cannot be both divisible by 3 , we may assume that $m \equiv 1$ or $m \equiv 2$. In view of $(*)$ we compute the reduced homology of $G$ of degree $|V|$. We can write $\varepsilon(G)=\varepsilon\left(\left\{x_{1}, x_{2}\right\}, \ldots,\left\{x_{m}, x_{1}\right\},\left\{x_{1}, y_{2}\right\},\left\{y_{2}, y_{3}\right\}, \ldots,\left\{y_{n-1}, y_{n}\right\},\left\{y_{n}, x_{1}\right\} ; V\right)=$ $E_{1} \cup E_{2}$, where $E_{1}=\varepsilon\left(\left\{x_{2}, x_{3}\right\}, \ldots,\left\{x_{m}, x_{1}\right\},\left\{x_{1}, y_{2}\right\}, \ldots,\left\{y_{n-1}, y_{n}\right\},\left\{y_{n}, x_{1}\right\} ; V\right)$ and $E_{2}=\varepsilon\left(\left\{x_{1}, x_{2}\right\} ; V\right)$. We have that $E_{1}=\varepsilon\left(L_{m} \cup C_{n}\right)$, where $L_{m}: x_{2} x_{3} \ldots x_{m} x_{1}$. The intersection of these simplicial complexes is $E_{1} \cap E_{2}=\varepsilon\left(\left\{x_{1}, x_{2}, x_{3}\right\},\left\{x_{1}, x_{2}, x_{3}\right.\right.$, $\left.x_{4}\right\}, \ldots,\left\{x_{1}, x_{2}, x_{m-1}, x_{m}\right\},\left\{x_{m}, x_{1}, x_{2}\right\},\left\{x_{1}, y_{2}, x_{2}\right\}, \ldots,\left\{y_{n-1}, y_{n}, x_{1}, x_{2}\right\},\left\{y_{n}, x_{1}\right.$, $\left.\left.x_{2}\right\} ; V\right)=\varepsilon\left(\left\{x_{3}\right\},\left\{x_{m}\right\},\left\{y_{2}\right\},\left\{y_{n}\right\},\left\{x_{4}, x_{5}\right\}, \ldots,\left\{x_{m-2}, x_{m-1}\right\},\left\{y_{3}, y_{4}\right\}, \ldots,\left\{y_{n-2}\right.\right.$, $\left.\left.y_{n-1}\right\} ; V \backslash\left\{x_{1}, x_{2}\right\}\right)$ (see [12, Lemma 3.4]).

By [12, Lemma 3.5], $\tilde{H}_{i}\left(E_{1} \cap E_{2}\right)=\tilde{H}_{i-4}(\varepsilon(H))$ for all $i$, where $H$ is the induced subgraph on $V \backslash\left\{x_{1}, x_{2}, x_{3}, x_{m}, y_{2}, y_{n}\right\}$. We have $\tilde{H}_{i}\left(E_{1} \cap E_{2}\right)=\tilde{H}_{i-4}\left(\varepsilon\left(L_{m-4} \cup L_{n-3}\right)\right)$ for all $i$. Since $E_{2}$ is a simplex, $\tilde{H}_{i}\left(E_{2}\right)=0$ for all $i$.

Case 1.1. Let $m \equiv 2$.

By Theorem C, $\tilde{H}_{i}\left(E_{1} \cap E_{2}\right)=0$ for any $i$, since $m-4 \equiv 1$. Applying Corollary 3.2 to the path $L_{m-2}: x_{2} x_{3} \ldots x_{m-1}$, we get that, for all $i, \tilde{H}_{i}\left(E_{1}\right)=\tilde{H}_{i-\frac{2(m-2)}{3}}\left(\varepsilon\left(L_{2} \cup\right.\right.$ $\left.C_{n}\right)$ ), where $L_{2}: x_{1} x_{m}$. If we apply Lemma 3.1 once again for $v=x_{m}$ (and $u=x_{1}$, so that $\left.N(u)=\left\{x_{m}, y_{2}, y_{n}\right\}\right)$, we then obtain $\tilde{H}_{i}\left(E_{1}\right)=\tilde{H}_{i-\frac{2(m-2)}{3}-3}\left(\varepsilon\left(L_{n-3}\right)\right)$, for all $i$, where $L_{n-3}: y_{3} \ldots y_{n-1}$. In part $(a)$, we have $n \equiv 0$. Theorem $\mathrm{D}$ and $(*)$ show that $\tilde{H}_{i}\left(E_{1}\right) \neq 0$ if and only if $i=\frac{2|V|+1}{3}-2$. The Mayer-Vietoris sequence implies that $\tilde{H}_{i}(\varepsilon(G)) \neq 0$ if and only if $i=\frac{2|V|+1}{3}-2$. By $\left(^{*}\right)$ it follows that pd $I(G) \geq \frac{2|V|+1}{3}$, as claimed. In part $(b)$, we have $n \equiv 2$. Theorem $\mathrm{D}$ and $(*)$ show that $\tilde{H}_{i}\left(E_{1}\right) \neq 0$ if and only if $i=\frac{2|V|}{3}-2$. As above, it follows that $\tilde{H}_{i}(\varepsilon(G)) \neq 0$ if and only if $i=\frac{2|V|}{3}-2$. In view of $(*)$ we deduce that $p d I(G) \geq \frac{2|V|}{3}$, as claimed.

Case 1.2. Let $m \equiv 1$.

By Theorem C, $\tilde{H}_{i}\left(E_{1} \cap E_{2}\right)=\tilde{H}_{i-4-\frac{2(m-4)}{3}}\left(\varepsilon\left(L_{n-3}\right)\right)$ for any $i$. Moreover, applying Corollary 3.2 to the path $L_{m-1}: x_{2} x_{3} \ldots x_{m}$, we get that, for all $i, \tilde{H}_{i}\left(E_{1}\right)=$ $\tilde{H}_{i-\frac{2(m-1)}{3}}\left(\varepsilon\left(C_{n}\right)\right)$. In part $(a)$, we have $n \equiv 1$. Hence, by Theorem $\mathrm{C}, \tilde{H}_{i}\left(E_{1} \cap E_{2}\right)=$ 0 for any $i$, since $n-3 \equiv 1$. On the other hand, Theorem $\mathrm{B}$ and $\left(^{*}\right)$ show that $\tilde{H}_{i}\left(E_{1}\right) \neq 0$ if and only if $i=\frac{2|V|+1}{3}-2$. We deduce that $\tilde{H}_{i}(\varepsilon(G)) \neq 0$ if and only if $i=\frac{2|V|+1}{3}-2$. By $(*)$ it follows that $p d I(G) \geq \frac{2|V|+1}{3}$, as claimed. In part $(b)$, we have $n \equiv 0$. By Theorem $\mathrm{D}$ and $(*)$ it follows that $\tilde{H}_{i}\left(E_{1} \cap E_{2}\right) \neq 0$ if and only if $i=\frac{2|V|}{3}-2$, in which case the $i$ th homology module is equal to $K$. Theorem $\mathrm{B}$ and $\left(^{*}\right)$ show that $\tilde{H}_{i}\left(E_{1}\right) \neq 0$ if and only if $i=\frac{2|V|}{3}-2$ in which case it is equal to $K^{2}$. Thus the Mayer-Vietoris sequence implies that $\tilde{H}_{i}(\varepsilon(G)) \neq 0$ for $i=\frac{2|V|}{3}-2$. In view of $(*)$ we deduce that $p d I(G) \geq \frac{2|V|}{3}$, as claimed.

Case 2. Let $|V| \equiv 2$.

Case 2.1. $m \equiv 0$.

We have $n \equiv 0$. First assume that $n=m=3$. We first show that in this case $p d I(G) \geq 4$. We use the fact that $p d I(G)=c d I(G)$, (see [15, Theorem $1]$ ), where $c d$ denotes the local cohomological dimension, i.e., for any ideal $I$ of 
$\mathrm{R}, c d I$ is the maximum index $i$ for which the local cohomology module $H_{I}^{i}(R)$ (of $R$ with respect to $I$ ) does not vanish. We have that $I(G)=I \cap J$, where $I=\left(x_{1}, x_{2} x_{3}, y_{2} y_{3}\right)$ and $J=\left(x_{2}, x_{3}, y_{2}, y_{3}\right)$. It is well-known that, whenever an ideal is a complete intersection, its height is the only index for which the cohomology module of $R$ with respect to this ideal does not vanish (see [10, Proposition 3.8] together with [10, Theorem 4.4], or together with [8, Example 2, p. 414]). Since $I+J=\left(x_{1}, x_{2}, x_{3}, y_{2}, y_{3}\right)$ we thus have that $H_{I+J}^{i}(R) \neq 0$ if and only if $i=5$. We also have that $c d J=4$. In the Mayer-Vietoris sequence for local cohomology (see [10, Section 3)

$$
\ldots \rightarrow H_{I+J}^{4}(R) \rightarrow H_{I}^{4}(R) \oplus H_{J}^{4}(R) \rightarrow H_{I \cap J}^{4}(R) \rightarrow \ldots,
$$

the left term is zero, whereas the middle term is not. It follows that the right term is non zero, too. This implies that $p d I(G)=c d I(G) \geq 4$. On the other hand, by Lemma 2.1, the elements $x_{1} x_{2}, x_{2} x_{3}+x_{1} x_{3}, x_{1} y_{2}, x_{1} y_{3}+y_{2} y_{3}$ generate $I(G)$, up to radical, which shows that $\operatorname{ara} I(G) \leq 4$. It follows that $p d I(G)=\operatorname{ara} I(G)=4$, which proves the claim in this case.

Without loss of generality we can thus assume that $m \geq 6$. We can write $\varepsilon(G)=$ $E_{1} \cup E_{2}$, where $E_{1}=\varepsilon\left(\left\{x_{1}, x_{2}\right\},\left\{x_{3}, x_{4}\right\}, \ldots,\left\{x_{m}, x_{1}\right\},\left\{x_{1}, y_{2}\right\}, \ldots,\left\{y_{n}, x_{1}\right\} ; V\right)$ and $E_{2}=\varepsilon\left(\left\{x_{2}, x_{3}\right\} ; V\right)$. We have that $E_{1} \cap E_{2}=\varepsilon\left(\left\{x_{1}\right\},\left\{x_{4}\right\},\left\{x_{5}, x_{6}\right\}, \ldots\right.$, $\left.\left\{x_{m-1}, x_{m}\right\},\left\{y_{2}, y_{3}\right\}, \ldots,\left\{y_{n-1}, y_{n}\right\}\right)$. By [12, Lemma 3.5]) it follows that $\tilde{H}_{i}\left(E_{1} \cap\right.$ $\left.E_{2}\right)=\tilde{H}_{i-2}\left(\varepsilon\left(L_{m-4} \cup L_{n-1}\right)\right)$, for all $i$. We also have that $E_{1}=\varepsilon\left(H_{1}\right)$, where $H_{1}$ is the union of $C_{n}$ and the paths $x_{3} \ldots x_{m} x_{1}$ and $x_{1} x_{2}$. Applying Lemma 3.1 for $v=x_{2}$ (and $u=x_{1}$, so that $N(u)=\left\{x_{2}, x_{m}, y_{2}, y_{n}\right\}$, we obtain that, for all $i$, $\tilde{H}_{i}\left(E_{1}\right)=\tilde{H}_{i-4}\left(\varepsilon\left(L_{m-3} \cup L_{n-3}\right)\right)$. Thus, by Theorem $\mathrm{C}$, we deduce that, for all $i$, $\tilde{H}_{i}\left(E_{1}\right)=\tilde{H}_{i-4-\frac{2(n-3)}{3}}\left(\varepsilon\left(L_{m-3}\right)\right)$. According to Theorem $\mathrm{D}$ and $\left(^{*}\right)$ it is non zero if and only if $i=\frac{2|V|+2}{3}-2$. Applying Theorem C, Theorem D and $\left(^{*}\right)$ we also get that $\tilde{H}_{i}\left(E_{1} \cap E_{2}\right) \neq 0$ if and only if $i=\frac{2|V|+2}{3}-4$. By the Mayer-Vietoris sequence we conclude that $\tilde{H}_{i}(\varepsilon(G)) \neq 0$ for $i=\frac{2|V|+2}{3}-2$. In view of $\left(^{*}\right)$ we deduce that pd $I(G) \geq \frac{2|V|+2}{3}$, as claimed.

Case 2.2. Let $m \equiv 1$.

We have $n \equiv 2$. Consider the induced subgraph $H^{\prime}$ on $V \backslash\left\{y_{2}\right\}$. Then $H^{\prime}$ is the union of $C_{m}$ and the path $L_{n-1}: y_{3} \ldots y_{n} y_{1}$. Applying Corollary 3.2 to the path $L_{n-2}: y_{3} \ldots y_{n}$, we obtain $\tilde{H}_{i}\left(\varepsilon\left(H^{\prime}\right)\right)=\tilde{H}_{i-\frac{2(n-2)}{3}}\left(\varepsilon\left(C_{m}\right)\right)$, for all $i$. By Theorem $\mathrm{B}$ and $(*)$ it is non zero if and only if $i=\frac{2|V|-1^{3}}{3}-2$. In view of $(*)$ we deduce that pd $I(G) \geq \frac{2|V|-1}{3}$, as claimed.

Now we find an upper bound for the arithmetical rank in each case. In the rest of the proof, we will refer to the polynomials $q_{i}$ introduced in Propositions 2.2 2.3 and 2.4 in each case, the polynomial $q_{i}^{\prime}$ will be the one obtained from $q_{i}$ by replacing each variable $x_{j}$ with $y_{j}$.

In part $(a)$, for $m \equiv 2$, by Proposition 2.4 the sequence $A_{m}: q_{0}, \ldots, q_{\underline{2(m-2)}}$, generates $I\left(C_{m}\right)$, up to radical and by Proposition 2.2 the sequence $A_{n}: q_{0}^{\prime}, \ldots, q_{\frac{2 n}{3}-1}^{\prime}$, generates $I\left(C_{n}\right)$, up to radical. Since $I(G)=I\left(C_{m}\right)+I\left(C_{n}\right)$, the following sequence generates $I(G)$, up to radical: $B: q_{0}, \ldots, q_{\frac{2(m-2)}{3}}, q_{0}^{\prime}, \ldots, q_{\frac{2 n}{3}-1}^{\prime}$. This implies that 
$\operatorname{ara} I(G) \leq \frac{2|V|+1}{3}$

If $m \equiv 1$, then, by Proposition 2.3 the sequence $A_{m}: q_{0}, \ldots, q_{\underline{2(m-1)}}$ generates $I\left(C_{m}\right)$, up to radical and the sequence $A_{n}: q_{0}^{\prime}, \ldots, q_{\frac{2(n-1)}{3}}^{\prime}$ generates $I\left(C_{n}\right)$, up to radical. The summand $x_{1} x_{m}$ of $q_{1}$ divides the product of the monomials $q_{\frac{2(m-1)}{3}}=x_{m-1} x_{m}$ and $q_{0}^{\prime}=y_{1} y_{2}=x_{1} y_{2}$. Thus by Lemma 2.1 the sequence $B: q_{0}, q_{1}, q_{\frac{2(m-1)}{3}}+q_{0}^{\prime}, q_{2}, \ldots, q_{\frac{2(m-1)}{3}-1}, q_{1}^{\prime}, \ldots, q_{\underline{2(n-1)}}^{\prime}$ of length $\frac{2|V|+1}{3}$ generates $I(G)$ up to radical. This implies that ara $I(G) \leq \frac{2\left|\frac{3}{V}\right|+1}{3}$.

In part $(b)$, for $m \equiv 2$, according to Proposition 2.4 the sequence $A_{m}: q_{0}, \ldots, q_{\underline{2(m-2)}}$, generates $I\left(C_{m}\right)$, up to radical and the sequence $A_{n}: q_{0}^{\prime}, \ldots, q_{\frac{2(n-2)}{3}}^{\prime}$, generates $I\left(C_{n}\right)$, up to radical. The sequence $B$ formed by the union of these two sequences generates $I(G)$, up to radical. This implies that ara $I(G) \leq \frac{2|V|}{3}$.

If $m \equiv 1$, then, by Proposition 2.3 the sequence $A_{m}: q_{0}, \ldots, q_{\frac{2(m-1)}{3}}$ generates $I\left(C_{m}\right)$, up to radical and by Proposition 2.2 the sequence $A_{n}: q_{0}^{\prime}, \ldots, q_{\frac{2 n}{3}-1}^{\prime}$ generates $I\left(C_{n}\right)$, up to radical. Thus by Lemma 2.1 the sequence $B: q_{0}, q_{1}, q_{\frac{2(m-1)}{3}}+$ $q_{0}^{\prime}, q_{2}, \ldots, q_{\frac{2(m-1)}{3}-1}, q_{1}^{\prime}, \ldots, q_{\frac{2 n}{3}-1}^{\prime}$ of length $\frac{2|V|}{3}$, generates $I(G)$, up to radical. So $\operatorname{ara} I(G) \leq \frac{2|V|}{3}$.

In part $(c)$, if $m \equiv 0$, then consider the sequence $B: q_{0}, \ldots, q_{\frac{2 m}{3}-1}, q_{0}^{\prime}, \ldots, q_{\frac{2 n}{3}-1}^{\prime}$, where $A_{m}: q_{0}, \ldots, q_{\frac{2 m}{3}-1}$ generates $I\left(C_{m}\right)$ and $A_{n}: q_{0}^{\prime}, \ldots, q_{\frac{2 n}{3}-1}^{\prime}$ generates $I\left(C_{n}\right)$, up to radical, by Proposition 2.2. This implies that ara $I(G) \leq \frac{2|V|+2}{3}$.

If $m \equiv 1$, then, by Proposition 2.3 , the sequence $A_{m}: q_{0}, \ldots, q_{\frac{2(m-1)}{3}}$, generates $I\left(C_{m}\right)$, up to radical. By Proposition 2.4 , the sequence $A_{n}: q_{0}^{\prime}, \ldots, q_{\underline{2(n-2)}}^{\prime}$, generates $I\left(C_{n}\right)$, up to radical. Thus by Lemma 2.1 the sequence $B: q_{0}, q_{1}, q_{\frac{2(m-1)}{3}}+$ $q_{0}^{\prime}, q_{2}, \ldots, q_{\frac{2(m-1)}{3}-1}, q_{1}^{\prime}, \ldots, q_{\frac{2(n-2)}{3}}^{\prime}$, generates $I(G)$, up to radical. This implies that $\operatorname{ara} I(G) \leq \frac{2|V|-1}{3}$.

Theorem 3.4. Let $G$ be the graph formed by two cycles $C_{n}$ and $C_{m}$ with a path joining a vertex of $C_{n}$ to a vertex of $C_{m}$. Then the following hold:

(a) If $|V(G)| \equiv 1$, then pd $I(G)=\operatorname{ara} I(G)=\frac{2|V(G)|-2}{3}$, whenever $m \equiv 2, n \equiv 2$. Otherwise, pd $I(G)=\operatorname{ara} I(G)=\frac{2|V(G)|+1}{3}$.

(b) If $|V(G)| \equiv 0$, then pd $I(G)=\operatorname{ara} I(G)=\frac{2|V(G)|}{3}$.

(c) If $|V(G)| \equiv 2$ and $m, n \equiv 0$ or 1 , then $p d I(G)=\operatorname{ara} I(G)=\frac{2|V(G)|+2}{3}$. Otherwise, pd $I(G)=\operatorname{ara} I(G)=\frac{2|V(G)|-1}{3}$.

Proof. Let $V=V(G)$. Consider the labeling for $V$ such that $V\left(C_{n}\right)=\left\{y_{1}, y_{2}, \ldots\right.$, $\left.y_{n}\right\}, V\left(C_{m}\right)=\left\{x_{1}, x_{2}, \ldots, x_{m}\right\}$ and let $P: z_{0} z_{1} \ldots z_{k} z_{k+1}$ be the path in $G$, where $z_{0}=x_{1}$ and $z_{k+1}=y_{1}$. We compute the reduced homology of $G$ of degree $|V|$. Up to exchanging $m$ and $n$, we have the following cases. 
Case 1. Let $k \equiv 2$.

We can write $\varepsilon(G)=\varepsilon\left(\left\{x_{1}, x_{2}\right\}, \ldots,\left\{x_{m}, x_{1}\right\},\left\{y_{1}, y_{2}\right\}, \ldots,\left\{y_{n}, y_{1}\right\},\left\{x_{1}, z_{1}\right\},\left\{z_{1}, z_{2}\right\}\right.$, $\left.\ldots,\left\{z_{k-1}, z_{k}\right\},\left\{z_{k}, y_{1}\right\} ; V\right)=E_{1} \cup E_{2}$, where

$E_{1}=\varepsilon\left(\left\{x_{1}, x_{2}\right\}, \ldots,\left\{x_{m}, x_{1}\right\},\left\{y_{1}, y_{2}\right\}, \ldots,\left\{y_{n}, y_{1}\right\},\left\{x_{1}, z_{1}\right\},\left\{z_{1}, z_{2}\right\}, \ldots,\left\{z_{k-1}, z_{k}\right\}\right.$; $V)$ and $E_{2}=\varepsilon\left(\left\{z_{k}, y_{1}\right\} ; V\right)$. The intersection of these simplicial complexes is

$E_{1} \cap E_{2}=\varepsilon\left(\left\{y_{2}\right\},\left\{y_{n}\right\},\left\{z_{k-1}\right\},\left\{x_{1}, x_{2}\right\}, \ldots,\left\{x_{m}, x_{1}\right\},\left\{y_{3}, y_{4}\right\}, \ldots,\left\{y_{n-2}, y_{n-1}\right\}\right.$, $\left.\left\{x_{1}, z_{1}\right\},\left\{z_{1}, z_{2}\right\}, \ldots,\left\{z_{k-3}, z_{k-2}\right\} ; V\right)$ (see [12, Lemma 3.4]).

By [12, Lemma 3.5] it follows that

$$
\tilde{H}_{i}\left(E_{1} \cap E_{2}\right)=\tilde{H}_{i-3}\left(\varepsilon\left(H_{1} \cup L_{n-3}\right)\right),
$$

for all $i$, where $H_{1}$ is the induced subgraph on $V \backslash\left(V\left(C_{n}\right) \cup\left\{z_{k-1}, z_{k}\right\}\right)$. Applying Corollary 3.2 to the path $L_{k-2}: z_{1} \ldots z_{k-2}$, we have

$$
\tilde{H}_{i}\left(E_{1} \cap E_{2}\right)=\tilde{H}_{i-\frac{2(k-2)}{3}-3}\left(\varepsilon\left(C_{m} \cup L_{n-3}\right)\right),
$$

for all $i$. Since $E_{2}$ is a simplex, $\tilde{H}_{i}\left(E_{2}\right)=0$ for all $i$.

Applying Corollary 3.2 to the path $L_{k+1}: z_{0} \ldots z_{k}$, we have that, for all $i$,

$$
\tilde{H}_{i}\left(E_{1}\right)=\tilde{H}_{i-\frac{2(k+1)}{3}}\left(\varepsilon\left(L_{m-1} \cup C_{n}\right)\right) \text {. }
$$

Case 1.1 Let $n \equiv 1$.

By Theorem $\mathrm{C}$, since $n-3 \equiv 1$, we have that

$$
\tilde{H}_{i}\left(E_{1} \cap E_{2}\right)=0
$$

for all $i$. The Mayer-Vietoris sequence then implies that $\tilde{H}_{i}(\varepsilon(G))=\tilde{H}_{i}\left(E_{1}\right)$ for all $i$. Moreover, in view of Proposition $2.3, I\left(C_{n}\right)$ is generated, up to radical, by the sequence $A_{n}: q_{0}^{\prime}, \ldots, q_{\frac{2(n-1)}{3}}^{\prime}$.

Case 1.1.1 Let $m \equiv 1$ or $m \equiv 0$.

First suppose that $m \equiv 1$. Then $|V| \equiv 1$. From Theorem $\mathrm{C}$ we have that, for all $i$, $\tilde{H}_{i}\left(E_{1}\right)=\tilde{H}_{i-\frac{2(k+1)}{3}-\frac{2(m-1)}{3}}\left(\varepsilon\left(C_{n}\right)\right)$. In view of Theorem B and $\left(^{*}\right)$ it follows that $\tilde{H}_{i}\left(E_{1}\right) \neq 0$ if and only if $i=\frac{2|V|+1}{3}-2$. Thus $\tilde{H}_{i}(\varepsilon(G)) \neq 0$ for $i=\frac{2|V|+1}{3}-2$, which, by $(*)$, implies that $p d I(G) \geq \frac{2|V|+1}{3}$.

By Lemma 2.1, the sequence $B: q_{0}, q_{1}, q_{\frac{2(m-1)}{3}}+x_{1} z_{1}, q_{2}, \ldots, q_{\frac{2(m-1)}{3}-1}, z_{k} y_{1}, z_{k-1} z_{k}$ $+q_{0}^{\prime}, q_{1}^{\prime}, \ldots, q_{\frac{2(n-1)}{3}}^{\prime}, z_{2} z_{3}, z_{1} z_{2}+z_{3} z_{4}, \ldots, z_{k-3} z_{k-2}, z_{k-4} z_{k-3}+z_{k-2} z_{k-1}$ of length $\frac{2|V|+1}{3}$, generates $I(G)$, up to radical, where $A_{m}: q_{0}, \ldots, q_{\frac{2(m-1)}{3}}$ generates $I\left(C_{m}\right)$, up to radical by Proposition 2.3. Thus ara $I(G) \leq \frac{2|V|+1}{3}$.

Now suppose that $m \equiv 0$. Then $|V| \equiv 0$. From Theorem $\mathrm{C}$, Theorem B and (*) we deduce that $\tilde{H}_{i}\left(E_{1}\right) \neq 0$ if and only if $i=\frac{2|V|}{3}-2$. Thus $\tilde{H}_{i}(\varepsilon(G)) \neq 0$ for $i=\frac{2|V|}{3}-2$, which, by $(*)$, implies that $p d I(G) \geq \frac{2|V|}{3}$.

By Lemma 2.1, the sequence $B: q_{0}^{\prime}, q_{1}^{\prime}, q_{\frac{2(n-1)}{3}}^{\prime}+y_{1} z_{k}, q_{2}^{\prime}, \ldots, q_{\frac{2(n-1)}{3}-1}^{\prime}, z_{1} x_{1}, z_{1} z_{2}+$ $q_{0}, q_{1}, \ldots, q_{\frac{2 m}{3}-1}, z_{3} z_{4}, z_{2} z_{3}+z_{4} z_{5}, \ldots, z_{k-2} z_{k-1}, z_{k-3} z_{k-2}+z_{k-1} z_{k}$ of length $\frac{2|V|}{3}$, generates $I(G)$, up to radical, where $A_{m}: q_{0}, \ldots, q_{\frac{2 m}{3}}$ generates $I\left(C_{m}\right)$, up to radical by Proposition 2.2 Therefore, we have $p d I(G)=\operatorname{ara} I(G)=\frac{2|V|}{3}$.

Case 1.1.2 Let $m \equiv 2$.

In this case $|V| \equiv 2$. Consider the induced subgraph $H_{2}$ on $V \backslash\left\{z_{k}\right\}$. We have 
$H_{2}=H^{\prime} \cup C_{n}$, where $H^{\prime}$ is the induced subgraph on $V \backslash\left(V\left(C_{n}\right) \cup\left\{z_{k}\right\}\right)$. By Theorem A we have that, for all $i, \tilde{H}_{i}\left(\varepsilon\left(H_{2}\right)\right)=\tilde{H}_{i-\frac{2 n+1}{3}}\left(\varepsilon\left(H^{\prime}\right)\right)$. If we apply Corollary 3.2 along the path $L_{k-2}: z_{2} \ldots z_{k-1}$, and then Lemma 3.1 for $v=z_{1}$, we further get, for all $i, \tilde{H}_{i}\left(\varepsilon\left(C_{n} \cup H^{\prime}\right)\right)=\tilde{H}_{i-\frac{2 n+1}{3}-\frac{2(k-2)}{3}-3}\left(\varepsilon\left(L_{m-3}\right)\right)$, which, by Theorem $\mathrm{D}$ and $(*)$, is non zero in $i=\frac{2|V|-1}{3}-2$. By $\left(^{*}\right)$ this implies $p d I(G) \geq \frac{2|V|-1}{3}$.

By Lemma2.1, the sequence $B: z_{1} x_{1}, z_{1} z_{2}+q_{0}, q_{1}, \ldots, \underline{\underline{2(m-2)}}, z_{3} z_{4}, z_{2} z_{3}+z_{4} z_{5}, \ldots, z_{k-2} z_{k-1}, z_{k-3} z_{k-2}+$

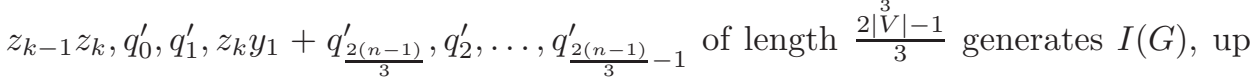
to radical, where the sequence $A_{n}: q_{0}^{\prime}, \ldots, q_{\frac{2(n-1)}{3}}^{\prime}$ generates $I\left(C_{n}\right)$, up to radical, by Proposition 2.3 This shows that ara $I(G) \leq \frac{2|V|-1}{3}$.

Case 1.2 Let $n \equiv 2$.

By Theorem C, since $n-3 \equiv 2$, we have that

$$
\tilde{H}_{i}\left(E_{1} \cap E_{2}\right)=\tilde{H}_{i-\frac{2(k-2)}{3}-3-\frac{2(n-3)-1}{3}}\left(\varepsilon\left(C_{m}\right)\right)
$$

for all $i$. Moreover, by Theorem A,

$$
\tilde{H}_{i}\left(E_{1}\right)=\tilde{H}_{i-\frac{2(k+1)}{3}-\frac{2 n-1}{3}}\left(\varepsilon\left(L_{m-1}\right)\right),
$$

for all $i$. Moreover, in view of Proposition 2.4 $I\left(C_{n}\right)$ is generated, up to radical, by the sequence $A_{n}: q_{0}^{\prime}, \ldots, q_{\frac{2(n-2)}{3}}^{\prime}$.

Case 1.2.1 Let $m \equiv 0$.

In this case $|V| \equiv 1$. In view of Theorem B and $\left(^{*}\right)$ we have that $\tilde{H}_{i}\left(E_{1} \cap E_{2}\right) \neq 0$ if and only if $i=\frac{2|V|+1}{3}-3$, in which case the homology group is $K^{2}$, and, according to Theorem $\mathrm{D}$ and $\left(^{*}\right)$, we have that $\tilde{H}_{i}\left(E_{1}\right) \neq 0$ if and only if $i=\frac{2|V|+1}{3}-3$, in which case the homology group is $K$. From the Mayer-Vietoris sequence it then follows that $\tilde{H}_{i}(\varepsilon(G)) \neq 0$ for $i=\frac{2|V|+1}{3}-2$, which, by $\left(^{*}\right)$, implies that $p d I(G) \geq \frac{2|V|+1}{3}$. The sequence $B: q_{0}^{\prime}, \ldots, q_{\frac{2(n-2)}{3}}^{\prime}, q_{0}, \ldots, q_{\frac{2 m}{3}-1}, z_{1} z_{2}, z_{0} z_{1}+z_{2} z_{3}, \ldots, z_{k-1} z_{k}, z_{k-2} z_{k-1}$ $+z_{k} z_{k+1}$ of length $\frac{2|V|+1}{3}$ generates $I(G)$, up to radical, by Lemma 2.1. where the sequence $A_{m}: q_{0}, \ldots, q_{\frac{2 m}{3}-1}$ generates $I\left(C_{m}\right)$, up to radical, by Proposition 2.2 This implies that ara $I(G) \leq \frac{2|V|+1}{3}$.

Case 1.2.2 Let $m \equiv 2$.

In this case $|V| \equiv 0$. In view of Theorem B and $\left(^{*}\right)$ we have that $\tilde{H}_{i}\left(E_{1} \cap E_{2}\right) \neq 0$ if and only if $i=\frac{2|V|}{3}-3$, and, according to Theorem $\mathrm{C}$, since $m-1 \equiv 1$, we have that $\tilde{H}_{i}\left(E_{1}\right)=0$ for all $i$. From the Mayer-Vietoris sequence it then follows that $\tilde{H}_{i}(\varepsilon(G)) \neq 0$ for $i=\frac{2|V|}{3}-2$, which, by $\left(^{*}\right)$, implies that $p d I(G) \geq \frac{2|V|}{3}$.

By Lemma 2.1, the sequence $B: q_{0}, \ldots, q_{\frac{2(m-2)}{3}}, q_{0}^{\prime}, \ldots, q_{\frac{2(n-2)}{3}}^{\prime}, z_{1} z_{2}, z_{0} z_{1}+z_{2} z_{3}, \ldots$, $z_{k-1} z_{k}, z_{k-2} z_{k-1}+z_{k} z_{k+1}$ of length $\frac{2|V|}{3}$ generates $I(G)$, up to radical, where the sequence $A_{m}: q_{0}, \ldots, q_{\frac{2(m-2)}{3}}$ generates $I\left(C_{m}\right)$, up to radical, by Proposition 2.4 This implies that ara $I(G) \leq \frac{2|V|}{3}$.

Case 1.3 Let $n \equiv m \equiv 0$. 
In this case $|V| \equiv 2$. In view of Theorem $\mathrm{C}$, since $n-3 \equiv 0$, we have that, for all $i$,

$$
\tilde{H}_{i}\left(E_{1} \cap E_{2}\right)=\tilde{H}_{i-\frac{2(k-2)}{3}-3-\frac{2(n-3)}{3}}\left(\varepsilon\left(C_{m}\right)\right),
$$

and by Theorem A, for all $i$,

$$
\tilde{H}_{i}\left(E_{1}\right)=\tilde{H}_{i-\frac{2(k+1)}{3}-\frac{2 n}{3}}\left(\varepsilon\left(L_{m-1}\right)\right) .
$$

According to Theorem B and $\left(^{*}\right)$ it follows that $\tilde{H}_{i}\left(E_{1} \cap E_{2}\right) \neq 0$ if and only if $i=\frac{2|V|+2}{3}-3$, in which case it is equal to $K^{2}$, and, in view of Theorem $\mathrm{D}$ and $\left.{ }^{*}\right), \tilde{H}_{i}\left(E_{1}\right) \neq 0$ if and only if $i=\frac{2|V|+2}{3}-3$, in which case it is equal to $K$. From the Mayer-Vietoris sequence it then follows that $\tilde{H}_{i}(\varepsilon(G)) \neq 0$ for $i=\frac{2|V|+2}{3}-2$, which, by $\left(^{*}\right)$, implies that $p d I(G) \geq \frac{2|V|+2}{3}$.

The sequence $B: q_{0}^{\prime}, \ldots, q_{\frac{2 n}{3}-1}^{\prime}, q_{0}, \ldots, q_{\frac{2 m}{3}-1}, z_{1} z_{2}, x_{1} z_{1}+z_{2} z_{3}, \ldots, z_{k-1} z_{k}, z_{k-2} z_{k-1}$ $+z_{k} y_{1}$, generates $I(G)$, up to radical, by Lemma 2.1, where $A_{m}: q_{0}, \ldots, q_{\frac{2 m}{3}-1}$ generates $I\left(C_{m}\right)$, up to radical, and $A_{n}: q_{0}^{\prime}, \ldots, q_{\frac{2 n}{3}-1}^{\prime}$ generates $I\left(C_{n}\right)$, up to radical, by Proposition 2.2. Therefore, we have ara $I(G) \leq \frac{2|V|+2}{3}$.

Case 2 Let $k \equiv 0$.

As in Case 1 , we can write $\varepsilon(G)=\varepsilon\left(\left\{x_{1}, x_{2}\right\}, \ldots,\left\{x_{m}, x_{1}\right\},\left\{y_{1}, y_{2}\right\}, \ldots,\left\{y_{n}, y_{1}\right\}\right.$, $\left.\left\{x_{1}, z_{1}\right\},\left\{z_{1}, z_{2}\right\}, \ldots,\left\{z_{k-1}, z_{k}\right\},\left\{z_{k}, y_{1}\right\} ; V\right)=E_{1} \cup E_{2}$, where $E_{1}=\varepsilon\left(\left\{x_{1}, x_{2}\right\}, \ldots,\left\{x_{1}, x_{m}\right\},\left\{y_{1}, y_{2}\right\}, \ldots,\left\{y_{1}, y_{n}\right\},\left\{x_{1}, z_{1}\right\}, \ldots,\left\{z_{k-1}, z_{k}\right\}\right)$ and $E_{2}=\varepsilon\left(\left\{z_{k}, y_{1}\right\}\right)$. If $k=0$, then $E_{1} \cap E_{2}=\varepsilon\left(\left\{x_{2}\right\},\left\{x_{m}\right\},\left\{y_{2}\right\},\left\{y_{n}\right\},\left\{x_{3}, x_{4}\right\}, \ldots,\left\{x_{m-2}, x_{m-1}\right\}\right.$, $\left.\left\{y_{3}, y_{4}\right\}, \ldots,\left\{y_{n-2}, y_{n-1}\right\} ; V \backslash\left\{x_{1}, y_{1}\right\}\right)$, so that, by [11, Lemma 3.5],

$$
\tilde{H}_{i}\left(E_{1} \cap E_{2}\right)=\tilde{H}_{i-4}\left(\varepsilon\left(L_{m-3} \cup L_{n-3}\right)\right),
$$

for all $i$. If $k \geq 3$, then $E_{1} \cap E_{2}=\varepsilon\left(\left\{z_{k-1}\right\},\left\{y_{2}\right\},\left\{y_{n}\right\},\left\{x_{1}, x_{2}\right\}, \ldots,\left\{x_{m}, x_{1}\right\},\left\{y_{3}, y_{4}\right\}\right.$, $\left.\ldots,\left\{y_{n-2}, y_{n-1}\right\},\left\{x_{1}, z_{1}\right\},\left\{z_{1}, z_{2}\right\}, \ldots\left\{z_{k-3}, z_{k-2}\right\} ; V \backslash\left\{z_{k}, y_{1}\right\}\right)$, so that, by [11, Lemma 3.5], $\tilde{H}_{i}\left(E_{1} \cap E_{2}\right)=\tilde{H}_{i-3}\left(\varepsilon\left(H^{\prime \prime} \cup L_{n-3}\right)\right)$, for all $i$, where $H^{\prime \prime}$ is the induced subgraph on $V \backslash\left(V\left(C_{n}\right) \cup\left\{z_{k-1}, z_{k}\right\}\right)$, i.e., it is the union of $C_{m}$ and the path $L_{k-1}: x_{1} z_{1} \ldots z_{k-2}$. If we apply Corollary 3.2 along the path $L_{k-3}: z_{2} \ldots z_{k-2}$ and then Lemma 3.1 for $v=z_{1}$, we deduce that, for all $i$,

$$
\tilde{H}_{i}\left(E_{1} \cap E_{2}\right)=\tilde{H}_{i-6-\frac{2(k-3)}{3}}\left(\varepsilon\left(L_{m-3} \cup L_{n-3}\right)\right),
$$

which is evidently also true for $k=0$. If $k=0$, we have that $E_{1}=\varepsilon\left(C_{m} \cup C_{n}\right)$, otherwise, if we apply Corollary 3.2 along the path $L_{k}: z_{1} \ldots z_{k}$, we obtain that, for all $i$,

$$
\tilde{H}_{i}\left(E_{1}\right)=\tilde{H}_{i-\frac{2 k}{3}}\left(C_{m} \cup C_{n}\right) \text {. }
$$

This equality is evidently also true for $k=0$. Since $E_{2}$ is a simplex, we also have that $\tilde{H}_{i}\left(E_{2}\right)=0$ for all $i$.

Case 2.1 Let $n \equiv 1$.

In view of Theorem $\mathrm{D}$ (for $m=3$ ) and of Theorem $\mathrm{C}$ (for $m \geq 4$ ), since $n-3 \equiv 1$, we have that $\tilde{H}_{i}\left(E_{1} \cap E_{2}\right)=0$ for all $i$, so that $\tilde{H}_{i}(\varepsilon(G))=\tilde{H}_{i}\left(E_{1}\right)$ for all $i$. Moreover, in view of Theorem A, for all $i, \tilde{H}_{i}\left(E_{1}\right)=\tilde{H}_{i-\frac{2 k}{3}-\frac{2 n+1}{3}}\left(\varepsilon\left(C_{m}\right)\right)$.

By Proposition 2.3, the sequence $A_{n}: q_{0}^{\prime}, q_{1}^{\prime}, \ldots, \frac{q_{2(n-1)}}{3}$ generates $I\left(C_{n}\right)$, up to radical. 
Let $m \equiv 1$. Then $|V| \equiv 2$. In view of Theorem B and $\left(^{*}\right), \tilde{H}_{i}\left(E_{1}\right) \neq 0$ if and only if $i=\frac{2|V|+2}{3}-2$. Hence $\tilde{H}_{i}(\varepsilon(G)) \neq 0$ for $i=\frac{2|V|+2}{3}-2$, which, by $(*)$, implies that pd $I(G) \geq \frac{2|V|+2}{3}$.

If $k=0$, then the sequence $q_{0}, q_{1}, q_{\frac{2(m-1)}{3}}+x_{1} y_{1}, q_{2}, \ldots, q_{\frac{2(m-1)}{3}-1}, q_{0}^{\prime}, \ldots, q_{\underline{2(n-1)}}^{\prime}$ of length $\frac{2|V|+2}{3}$, generates $I(G)$, up to radical, by Lemma 2.1, where the sequence $A_{m}: q_{0}, \ldots, q_{\frac{2(m-1)}{3}}$ generates $I\left(C_{m}\right)$, up to radical, by Proposition 2.3

If $k \geq 3$, then the sequence $B: q_{0}, q_{1}, q_{\frac{2(m-1)}{3}}+x_{1} z_{1}, q_{2}, \ldots, q_{\frac{2(m-1)}{3}-1}, z_{2} z_{3}, z_{1} z_{2}+$ $z_{3} z_{4}, \ldots, z_{k-1} z_{k}, z_{k-2} z_{k-1}+z_{k} y_{1}, q_{0}^{\prime}, \ldots, q_{\underline{2(n-1)}}^{\prime}$ of length $\frac{2|V|+2}{3}$, generates $I(G)$, up to radical. Hence we have ara $I(G) \leq \frac{\overline{2|V|+2}}{3}$.

Let $m \equiv 2$. Then $|V| \equiv 0$. In view of Theorem B and $\left(^{*}\right), \tilde{H}_{i}\left(E_{1}\right) \neq 0$ if and only if $i=\frac{2|V|}{3}-2$. Hence $\tilde{H}_{i}(\varepsilon(G)) \neq 0$ for $i=\frac{2|V|}{3}-2$, which, by $\left(^{*}\right)$, implies that pd $I(G) \geq \frac{2|V|}{3}$.

If $k=0$, then by Lemma 2.1 , the sequence $B: q_{0}^{\prime}, q_{1}^{\prime}, q_{\frac{2(n-1)}{3}}^{\prime}+x_{1} y_{1}, q_{2}^{\prime}, \ldots, q_{\frac{2(n-1)}{3}-1}^{\prime}$, $q_{0}, \ldots, q_{\underline{2(m-2)}}$, of length $\frac{2|V|}{3}$, generates $I(G)$ up to radical, where the sequence $A_{m}: q_{0}, \ldots, q_{\frac{2(m-2)}{3}}$ generates $I\left(C_{m}\right)$, up to radical, by Proposition 2.4 If $k \geq 3$, then the sequence $B: q_{0}^{\prime}, q_{1}^{\prime}, q_{\frac{2(n-1)}{3}}^{\prime}+y_{1} z_{k}, q_{2}^{\prime}, \ldots, q_{\frac{2(n-1)}{3}-1}^{\prime}, z_{1} z_{2}, x_{1} z_{1}+$ $z_{2} z_{3}, \ldots, z_{k-2} z_{k-1}, z_{k-3} z_{k-2}+z_{k-1} z_{k}, q_{0}, \ldots, q_{\underline{2(m-2)}}$, of length $\frac{2|V|}{3}$, generates $I(G)$ up to radical. This implies that ara $I(G) \leq \frac{2|V|^{3}}{3}$.

Let $m \equiv 0$. Then $|V| \equiv 1$. In view of Theorem B and $\left(^{*}\right), \tilde{H}_{i}\left(E_{1}\right) \neq 0$ if and only if $i=\frac{2|V|+1}{3}-2$. Hence $\tilde{H}_{i}(\varepsilon(G)) \neq 0$ for $i=\frac{2|V|+1}{3}-2$, which, by $\left(^{*}\right)$, implies that pd $I(G) \geq \frac{2|V|+1}{3}$.

If $k=0$, then the sequence $B: q_{0}^{\prime}, q_{1}^{\prime}, q_{\frac{2(n-1)}{3}}^{\prime}+x_{1} y_{1}, q_{2}^{\prime}, \ldots, q_{\frac{2(n-1)}{3}-1}^{\prime}, q_{0}, \ldots, q_{\frac{2 m}{3}-1}$ of length $\frac{2|V|+1}{3}$, generates $I(G)$, up to radical, by Lemma 2.1. where the sequence $A_{m}: q_{0}, \ldots, q_{\frac{2 m}{3}-1}$ generates $I\left(C_{m}\right)$, up to radical, by Proposition 2.2

If $k \geq 3$, then the sequence $B: q_{0}^{\prime}, q_{1}^{\prime}, q_{\frac{2(n-1)}{3}}^{\prime}+y_{1} z_{k}, q_{2}^{\prime}, \ldots, q_{\frac{2(n-1)}{3}-1}^{\prime}, z_{1} z_{2}, x_{1} z_{1}+$ $z_{2} z_{3}, \ldots, z_{k-2} z_{k-1}, z_{k-3} z_{k-2}+z_{k-1} z_{k}, q_{0}, \ldots, q_{\frac{2 m}{3}-1}$ of length $\frac{2|V|+1}{3}$, generates $I(G)$, up to radical, by Lemma 2.1. This shows that ara $I(G) \leq \frac{2|V|+1}{3}$.

Case 2.2 Let $n \equiv 2$ and $m \equiv 0$.

In this case $|V| \equiv 2$. In view of Theorem $\mathrm{C}$, Theorem $\mathrm{D}$ and $\left({ }^{*}\right), \tilde{H}_{i}\left(E_{1} \cap E_{2}\right) \neq 0$ if and only if $i=\frac{2|V|-1}{3}-2$, in which case the homology group is $K$. Moreover, in view of Theorem $\mathrm{A}, \tilde{H}_{i}\left(E_{1}\right)=\tilde{H}_{i-\frac{2 k}{3}-\frac{2 m}{3}}\left(\varepsilon\left(C_{n}\right)\right)$, for all $i$. In view of Theorem $\mathrm{B}$ and $\left(^{*}\right), \tilde{H}_{i}\left(E_{1}\right) \neq 0$ if and only if $i=\frac{2|V|-1}{3}-2$, in which case the homology group is $K^{2}$. Hence $\tilde{H}_{i}(\varepsilon(G)) \neq 0$ for $i=\frac{2|V|-1}{3}-2$, which, by $\left(^{*}\right)$, implies that pd $I(G) \geq \frac{2|V|-1}{3}$.

Let $k=0$. The sequence $B: x_{1} y_{1}, q_{0}+q_{0}^{\prime}, q_{1}, \ldots, q_{\frac{2 m}{3}-1}, q_{1}^{\prime}, \ldots, q_{\frac{2(n-2)}{3}}^{\prime}$ of length $\frac{2|V|-1}{3}$ generates $I(G)$, up to radical, by Lemma 2.1] where the sequence $A_{m}$ : $q_{0}, q_{1}, \ldots, q_{\frac{2 m}{3}-1}$ generates $I\left(C_{m}\right)$, up to radical, by Proposition 2.2 
Let $k \geq 3$. The sequence $B: x_{1} z_{1}, q_{0}+z_{1} z_{2}, q_{1}, \ldots, q_{\frac{2 m}{3}-1}, z_{3} z_{4}, z_{2} z_{3}+z_{4} z_{5}, \ldots$, $z_{k-3} z_{k-2}, z_{k-4} z_{k-3}+z_{k-2} z_{k-1}, z_{k} y_{1}, z_{k-1} z_{k}+q_{0}^{\prime}, q_{1}^{\prime}, \ldots, q_{\underline{2(n-2)}}^{\prime}$ of length $\frac{2|V|-1}{3}$ generates $I(G)$, up to radical. Hence, in view of $\left(^{*}\right)$, we conclude that ara $I(G) \leq$ $\frac{2|V|-1}{3}$.

Case 2.3 Let $n \equiv m \equiv 2$.

In this case $|V| \equiv 1$. Consider the induced subgraph $H_{3}$ on $V \backslash\left\{x_{2}\right\}$. Applying Corollary 3.2 to the path $L_{m+k-2}: x_{3} x_{4} \ldots x_{1} z_{1} \ldots z_{k-1}\left(L_{m-2}: x_{3} x_{4} \ldots x_{1}\right.$, if $k=0$ ) and Lemma 3.1 for $v=z_{k}\left(u=y_{1}\right)$, we obtain that, for all $i, \tilde{H}_{i}\left(\varepsilon\left(H_{3}\right)\right)=$ $\tilde{H}_{i-\frac{2(m+k-2)}{3}-3}\left(\varepsilon\left(L_{n-3}\right)\right)$, which, by Theorem $\mathrm{D}$ and $\left(^{*}\right)$, is non zero in $i=\frac{2|V|-2}{3}-2$. So $\tilde{H}_{\underline{2|V|-2}-2}(\varepsilon(G)) \neq 0$ and by $\left(^{*}\right)$ we have $p d I(G) \geq \frac{2|V|-2}{3}$.

If $k=0$, then, by Lemma 2.1] the sequence $B: x_{1} y_{1}, q_{0}+q_{0}^{\prime}, q_{1}, \ldots, q_{\frac{2(m-2)}{3}}, q_{1}^{\prime}, \ldots$, $q_{\frac{2(n-2)}{3}}^{\prime}$ generates $I(G)$, up to radical, where the sequence $A_{m}: q_{0}, \ldots, q_{\frac{2(m-2)}{3}}$ generates $I\left(C_{m}\right)$, up to radical, and the sequence $A_{n}: q_{0}^{\prime}, \ldots, q_{\frac{2(n-2)}{3}}^{\prime}$ generates $I\left(C_{n}\right)$, up to radical, by Proposition 2.4

If $k \geq 3$, then, by Lemma 2.1, the sequence $B: z_{k} y_{1}, z_{k-1} z_{k}+q_{0}^{\prime}, q_{1}^{\prime}, \ldots, q_{\underline{2(n-2)}}^{\prime}, z_{0} z_{1}$, $z_{1} z_{2}+q_{0}, q_{1}, \ldots, q_{\frac{2(m-2)}{3}}, z_{3} z_{4}, z_{2} z_{3}+z_{4} z_{5}, \ldots, z_{k-3} z_{k-2}, z_{k-4} z_{k-3}+z_{k-2} z_{k-1}$ generates $I(G)$, up to radical. Hence we have ara $I(G) \leq \frac{2|V|-2}{3}$.

Case 2.4 Let $n \equiv m \equiv 0$.

In this case $|V| \equiv 0$. First assume that $n=m=3$. We have that $I(G)=I \cap J$, where $I=I(G)+\left(x_{1} y_{1} z_{3} z_{6} \ldots z_{k}\right)$ and $J=\left(x_{2}, x_{3}, y_{2}, y_{3}, z_{1}, z_{2}, z_{4}, z_{5}, \ldots, z_{k-5}, z_{k-4}\right.$, $\left.z_{k-2}, z_{k-1}\right)$. Since $J$ is a complete intersection ideal, we have that $c d J=4+\frac{2 k}{3}$. Moreover, $I+J=\left(x_{1} y_{1} z_{3} z_{6} \ldots z_{k}, x_{2}, x_{3}, y_{2}, y_{3}, z_{1}, z_{2}, z_{4}, z_{5}, \ldots, z_{k-5}, z_{k-4}, z_{k-2}, z_{k-1}\right)$. Since $I+J$ has grade equal to $5+\frac{2 k}{3}$, by [5]. Theorem 6.2.7] we have $H_{I+J}^{i}(R) \neq 0$ in $i=5+\frac{2 k}{3}$ and $H_{I+J}^{i}(R)=0$ for any $i<5+\frac{2 k}{3}$. In the Mayer-Vietoris sequence for local cohomology (see [10], Section 3)

$$
\ldots \rightarrow H_{I+J}^{4+\frac{2 k}{3}}(R) \rightarrow H_{I}^{4+\frac{2 k}{3}}(R) \oplus H_{J}^{4+\frac{2 k}{3}}(R) \rightarrow H_{I \cap J}^{4+\frac{2 k}{3}}(R) \rightarrow \ldots
$$

the left term is zero, whereas the middle term is not. It follows that the right term is non zero, too. This implies that $p d I(G)=c d I(G) \geq 4+\frac{2 k}{3}=\frac{2|V|}{3}$.

So without loss of generality we may assume that $n>3$. Then from Theorem $\mathrm{C}$, since $m-3 \equiv 0$, we have that $\tilde{H}_{i}\left(E_{1} \cap E_{2}\right)=\tilde{H}_{i-6-\frac{2(k-3)}{3}-\frac{2(m-3)}{3}}\left(\varepsilon\left(L_{n-3}\right)\right)$, for all $i$. Hence, in view of Theorem $\mathrm{D}$ and $\left(^{*}\right)$, we have that $\tilde{H}_{i}\left(E_{1} \cap E_{2}\right) \neq 0$ only if $i=\frac{2|V|}{3}-2$, in which case this homology group is $K$. In view of Theorem A, Theorem B and $\left(^{*}\right)$ we also have that $\tilde{H}_{i}\left(E_{1}\right) \neq 0$ only if $i=\frac{2|V|}{3}-2$, in which case this homology group is $K^{2}$. The Mayer-Vietoris sequence shows that $\tilde{H}_{i}(\varepsilon(G)) \neq 0$ in $i=\frac{2|V|}{3}-2$. Thus in view of $(*)$ we deduce that $p d I(G) \geq \frac{2|V|}{3}$.

If $k=0$, then, by Lemma 2.1, the sequence $B: x_{1} y_{1}, q_{0}+q_{0}^{\prime}, q_{1}, \ldots, q_{\frac{2 m}{3}-1}, q_{1}^{\prime}, \ldots$, $q_{\frac{2 n}{3}-1}^{\prime}$ of length $\frac{2|V|}{3}$ generates $I(G)$, up to radical, where the sequence $A_{m}$ : $q_{0}, \ldots, q_{\frac{2 m}{3}-1}$ generates $I\left(C_{m}\right)$, up to radical, and the sequence $A_{n}: q_{0}^{\prime}, \ldots, q_{\frac{2 n}{3}-1}^{\prime}$ generates $I\left(C_{n}\right)$, up to radical, by Proposition 2.2

If $k \geq 3$, then, by Lemma 2.1 the sequence $B: z_{1} x_{1}, z_{1} z_{2}+q_{0}, q_{1}, \ldots, q_{\frac{2 m}{3}-1}, z_{3} z_{4}$, 
116IARGHERITA BARILE, DARIUSH KIANI, FATEMEH MOHAMMADI, AND SIAMAK YASSEMI

$z_{2} z_{3}+z_{4} z_{5}, \ldots, z_{k-3} z_{k-2}, z_{k-4} z_{k-3}+z_{k-2} z_{k-1}, z_{k} y_{1}, z_{k-1} z_{k}+q_{0}^{\prime}, q_{1}^{\prime}, \ldots, q_{\frac{2 n}{3}-1}^{\prime}$ of length $\frac{2|V|}{3}$ generates $I(G)$, up to radical. We thus have ara $I(G) \leq \frac{2|V|}{3}$.

Case 3 Let $k \equiv 1$.

We can write $\varepsilon(G)=\varepsilon\left(\left\{x_{1}, x_{2}\right\}, \ldots,\left\{x_{m}, x_{1}\right\},\left\{y_{1}, y_{2}\right\}, \ldots,\left\{y_{n}, y_{1}\right\},\left\{x_{1}, z_{1}\right\},\left\{z_{1}, z_{2}\right\}\right.$, $\left.\ldots,\left\{z_{k-1}, z_{k}\right\},\left\{z_{k}, y_{1}\right\} ; V\right)=E_{1} \cup E_{2}$, where

$E_{1}=\varepsilon\left(\left\{x_{2}, x_{3}\right\}, \ldots,\left\{x_{m}, x_{1}\right\},\left\{y_{1}, y_{2}\right\}, \ldots,\left\{y_{n}, y_{1}\right\},\left\{x_{1}, z_{1}\right\},\left\{z_{1}, z_{2}\right\}, \ldots,\left\{z_{k-1}, z_{k}\right\}\right.$, $\left.\left\{z_{k}, y_{1}\right\} ; V\right)$ and $E_{2}=\varepsilon\left(\left\{x_{1}, x_{2}\right\} ; V\right)$. We have that $E_{1}=\varepsilon\left(L_{m+k} \cup C_{n}\right)$, where $L_{m+k}: x_{2} \ldots x_{m} x_{1} z_{1} \ldots z_{k}$.

If $m=3$, then, by [12, Lemma 3.4], $E_{1} \cap E_{2}=\varepsilon\left(\left\{x_{3}\right\},\left\{z_{1}\right\},\left\{z_{2}, z_{3}\right\}, \ldots,\left\{z_{k}, y_{1}\right\}\right.$, $\left.\left\{y_{1}, y_{2}\right\}, \ldots,\left\{y_{n}, y_{1}\right\} ; V\right)$, so that, by [12, Lemma 3.5],

$$
\tilde{H}_{i}\left(E_{1} \cap E_{2}\right)=\tilde{H}_{i-2}\left(\varepsilon\left(H_{4}\right)\right),
$$

for all $i$, where $H_{4}$ is the induced subgraph on $V \backslash\left(V\left(C_{m}\right) \cup\left\{z_{1}\right\}\right)$, i.e., the union of $C_{n}$ and the path $L_{k}: z_{2} \ldots y_{1}$. If $m \geq 4$, then $E_{1} \cap E_{2}=\varepsilon\left(\left\{x_{3}\right\},\left\{x_{m}\right\},\left\{z_{1}\right\},\left\{x_{4}, x_{5}\right\}\right.$, $\left.\ldots,\left\{x_{m-2}, x_{m-1}\right\},\left\{z_{2}, z_{3}\right\}, \ldots,\left\{z_{k}, y_{1}\right\},\left\{y_{1}, y_{2}\right\}, \ldots,\left\{y_{n}, y_{1}\right\} ; V\right)$, so that, by [12, Lemma 3.5],

$$
\tilde{H}_{i}\left(E_{1} \cap E_{2}\right)=\tilde{H}_{i-3}\left(\varepsilon\left(L_{m-4} \cup H_{4}\right),\right.
$$

for all $i$. Since $E_{2}$ is a simplex, $\tilde{H}_{i}\left(E_{2}\right)=0$ for all $i$.

Case 3.1 Let $n \equiv 1$.

The sequence $A_{n}: q_{0}^{\prime}, \ldots, q_{\frac{2(n-1)}{3}}^{\prime}$ generates $I\left(C_{n}\right)$, up to radical, by Proposition 2.2

Case 3.1.1 Let $m \equiv 0$ or $m \equiv 2$.

First let $m \equiv 0$. Then $|V| \equiv 2$. If we apply Corollary 3.2 to the path $L_{m+k-1}$ : $x_{2} \ldots x_{m} x_{1} \ldots z_{k-1}$, and then Lemma 3.1 for $v=z_{k}$ we get, that, for all $i, \tilde{H}_{i}\left(E_{1}\right)=$ $\tilde{H}_{i-\frac{2(m+k-1)}{3}-3}\left(\varepsilon\left(L_{n-3}\right)\right)$, which is zero for all $i$ by Theorem $\mathrm{D}$ and $\left(^{*}\right)$. If $m \geq 6$, applying Theorem $\mathrm{C}(m-4 \equiv 2)$ and Corollary 3.2 to $E_{1} \cap E_{2}$ along the path $L_{k-1}: z_{2} \ldots z_{k}$, we deduce that, for all $i$,

$$
\tilde{H}_{i}\left(E_{1} \cap E_{2}\right)=\tilde{H}_{i-\frac{2(m-4)-1}{3}-\frac{2(k-1)}{3}-3}\left(\varepsilon\left(C_{n}\right)\right),
$$

which is also true for $m=3$.

By Theorem B and $(*), \tilde{H}_{\frac{2|V|+2}{3}-3}\left(E_{1} \cap E_{2}\right) \neq 0$. So by the Mayer-Vietoris sequence $\tilde{H}_{\frac{2|V|+2}{3}-2}(\varepsilon(G)) \neq 0$ and in view of $(*)$ we conclude that $p d I(G) \geq \frac{2|V|+2}{3}$.

By Lemma 2.1, $B: q_{0}^{\prime}, \ldots, q_{\frac{2(n-1)}{3}}^{\prime}, x_{1} z_{1}, z_{1} z_{2}+q_{0}, q_{1}, \ldots, q_{\frac{2 m}{3}-1}, z_{3} z_{4}, z_{2} z_{3}+z_{4} z_{5}$, $\ldots, z_{k-1} z_{k}, z_{k-2} z_{k-1}+z_{k} y_{1}$ of length $\frac{2|V|+2}{3}$ generates $I(G)$, up to radical, where sequence $A_{m}: q_{0}, \ldots, q_{\frac{2 m}{3}-1}$ generates $I\left(C_{m}\right)$, up to radical, by Proposition 2.2 Therefore, we have ara $I(G) \leq \frac{2|V|+2}{3}$.

Now let $m \equiv 2$. In this case $|V| \equiv 1$, and $m+k \equiv 0$. Moreover, by Theorem $\mathrm{C}$, since $m-4 \equiv 1$, we have that $\tilde{H}_{i}\left(E_{1} \cap E_{2}\right)=0$ for all $i$. Hence $\tilde{H}_{i}(\varepsilon(G))=\tilde{H}_{i}\left(E_{1}\right)$ for all $i$. Applying Corollary 3.2 to the path $L_{m+k}: x_{2} x_{3} \ldots x_{1} z_{1} \ldots z_{k}$ we obtain that, for all $i, \tilde{H}_{i}\left(E_{1}\right)=\tilde{H}_{i-\frac{2(m+k)}{3}}\left(\varepsilon\left(C_{n}\right)\right)$. By Theorem B and $(*), \tilde{H}_{i}\left(E_{1}\right) \neq 0$ in $i=\frac{2|V|+1}{3}-2$. Thus by $\left(^{*}\right)$ we have $p d I(G) \geq \frac{2|V|+1}{3}$.

By Lemma 2.1, the sequence $B: x_{1} z_{1}, q_{0}+z_{1} z_{2}, q_{1}, \ldots, q_{\frac{2(m-2)}{3}}, z_{3} z_{4}, z_{2} z_{3}+z_{4} z_{5}, \ldots, z_{k-1} z_{k}, z_{k-2} z_{k-1}+$ 
$z_{k} y_{1}, q_{0}^{\prime}, \ldots, q_{\frac{2(n-1)}{3}}^{\prime}$ of length $\frac{2|V|+1}{3}$ generates $I(G)$, up to radical, where the sequence $A_{m}: \frac{q_{0}^{\prime}}{\prime}, \ldots, q_{\frac{2(m-2)}{3}}^{\prime}$ generates $I\left(C_{m}\right)$, up to radical, by Proposition 2.4 Thus $\operatorname{ara} I(G) \leq \frac{2|V|+1}{3}$.

Case 3.1.2 Let $m \equiv 1$.

In this case $|V| \equiv 0$. Consider the induced subgraph $H_{5}$ on $V \backslash\left\{z_{k}\right\}$. We have, for all $i, \tilde{H}_{i}\left(\varepsilon\left(H_{5}\right)\right)=\tilde{H}_{i}\left(H^{\prime \prime \prime} \cup C_{n}\right)$, where $H^{\prime \prime \prime}$ is the induced subgraph on $V \backslash\left(V\left(C_{n}\right) \cup\left\{z_{k}\right\}\right)$, i.e., the union of $C_{m}$ and the path $L_{k}: x_{1} z_{1} \ldots z_{k-1}$. Applying Theorem A and then Corollary 3.2 to $H^{\prime \prime \prime}$ along the path $L_{k-1}: z_{1} \ldots z_{k-1}$ we have $\tilde{H}_{i}\left(\varepsilon\left(H_{5}\right)\right)=\tilde{H}_{i-\frac{2 n+1}{3}-\frac{2(k-1)}{3}}\left(C_{m}\right)$, for all $i$, and this homology group, by Theorem $\mathrm{B}$ and $\left(^{*}\right)$, is non zero in $i=\frac{2|V|}{3}-2$. So $\tilde{H}_{\frac{2|V|}{3}-2}\left(\varepsilon\left(H_{5}\right)\right) \neq 0$. In view of $(*)$ we deduce that $p d I(G) \geq \frac{2|V|}{3}$.

The sequence $B: q_{0}, q_{1}, q_{\frac{2(m-1)}{3}}+z_{1} x_{1}, \ldots, q_{\frac{2(m-1)}{3}-1}, q_{0}^{\prime}, q_{1}^{\prime}, q_{\frac{2(n-1)}{3}}^{\prime}+z_{k} y_{1}, \ldots$,

$q_{\frac{2(n-1)}{3}-1}^{\prime}, z_{2} z_{3}, z_{1} z_{2}+z_{3} z_{4}, \ldots, z_{k-2} z_{k-1}, z_{k-3} z_{k-2}+z_{k-1} z_{k}$ of length $\frac{2|V|}{3}$, generates $I(G)$, up to radical, by Lemma 2.1 where the sequence $A_{m}: q_{0}, \ldots, q_{2(m-1)}$ generates $I\left(C_{m}\right)$, up to radical, by Proposition 2.3. Therefore, we have that $\operatorname{ara} I(G) \leq \frac{2|V|}{3}$.

Case 3.2 Let $m \equiv 2$, and $n \equiv 0$ or 2 .

In this case $m+k \equiv 0$. Applying Corollary 3.2 to the path $L_{m+k}: x_{2} x_{3} \ldots x_{1} z_{1} \ldots z_{k}$ we obtain that, for all $i, \tilde{H}_{i}\left(E_{1}\right)=\tilde{H}_{i-\frac{2(m+k)}{3}}\left(\varepsilon\left(C_{n}\right)\right)$.

Moreover, the sequence $A_{m}: q_{0}, \ldots, q_{\frac{2(m-2)}{3}}$ generates $I\left(C_{m}\right)$, up to radical, by Proposition 2.4.

First let $n \equiv 0$. Then $|V| \equiv 0$ and, by Theorem B and $\left.{ }^{*}\right), \tilde{H}_{i}\left(E_{1}\right) \neq 0$ in $i=\frac{2|V|}{3}-2$. Thus by $(*)$ we have $p d I(G) \geq \frac{2|V|}{3}$.

By Lemma 2.1, the sequence $B: x_{1} z_{1}, q_{0}+z_{1} z_{2}, q_{1}, \ldots, q_{\frac{2(m-2)}{3}}, z_{3} z_{4}, z_{2} z_{3}+z_{4} z_{5}, \ldots, z_{k-1} z_{k}, z_{k-2} z_{k-1}+$ $z_{k} y_{1}, q_{0}^{\prime}, q_{1}^{\prime}, \ldots, q_{\frac{2 n}{3}-1}^{\prime}$ of length $\frac{2|V|}{3}$ generates $I(G)$, up to radical, where the sequence $A_{n}: q_{0}^{\prime}, \ldots, q_{\frac{2 n}{3}-1}^{\prime}$ generates $I\left(C_{n}\right)$, up to radical by Proposition 2.2. Thus ara $I(G) \leq \frac{2|V|}{3}$.

If $n \equiv 2$, then $|V| \equiv 2$ and, by Theorem B and $\left(^{*}\right), \tilde{H}_{i}\left(E_{1}\right) \neq 0$ in $i=\frac{2|V|-1}{3}-2$. Thus by $(*)$ we have $p d I(G) \geq \frac{2|V|-1}{3}$.

By Lemma 2.1] the sequence $B: q_{0}, \ldots, q_{\frac{2(m-2)}{3}}, z_{k} y_{1}, z_{k-1} z_{k}+q_{0}^{\prime}, q_{1}^{\prime}, \ldots, q_{\frac{2(n-2)}{3}}^{\prime}$, $z_{1} z_{2}, x_{1} z_{1}+z_{2} z_{3}, \ldots, z_{k-3} z_{k-2}, z_{k-4} z_{k-3}+z_{k-2} z_{k-1}$ of length $\frac{2|V|-1}{3}$ generates $I(G)$, up to radical, where the sequence $A_{n}: q_{0}^{\prime}, \ldots, q_{\underline{2(n-2)}}^{\prime}$ generates $I\left(C_{n}\right)$, up to radical by Proposition 2.4. Thus ara $I(G) \leq \frac{2|V|-1}{3}$.

Case 3.3 Let $n \equiv m \equiv 0$.

In this case $|V| \equiv 1$. As in Case 1 , we can write $\varepsilon(G)=E_{1} \cup E_{2}$, where $E_{1}=$ $\varepsilon\left(\left\{x_{1}, x_{2}\right\}, \ldots,\left\{x_{m}, x_{1}\right\},\left\{y_{1}, y_{2}\right\}, \ldots,\left\{y_{n}, y_{1}\right\},\left\{x_{1}, z_{1}\right\},\left\{z_{1}, z_{2}\right\}, \ldots,\left\{z_{k-1}, z_{k}\right\} ; V\right)$ and $E_{2}=\varepsilon\left(\left\{z_{k}, y_{1}\right\} ; V\right)$.

Applying Corollary 3.2 to the path $L_{k-1}: x_{1} z_{1} \ldots z_{k-2}$, we have that, for all $i$, 
18IARGHERITA BARILE, DARIUSH KIANI, FATEMEH MOHAMMADI, AND SIAMAK YASSEMI

$\tilde{H}_{i}\left(E_{1} \cap E_{2}\right)=\tilde{H}_{i-\frac{2(k-1)}{3}-3}\left(\varepsilon\left(L_{m-1} \cup L_{n-3}\right)\right)$. Theorem $\mathrm{C}(m-1 \equiv 2)$, Theorem $\mathrm{D}$ and $\left(^{*}\right)$ show that $\tilde{H}_{i}\left(E_{1} \cap E_{2}\right) \neq 0$ only if $i=\frac{2|V|+1}{3}-3$. Since $E_{2}$ is a simplex, $\tilde{H}_{i}\left(E_{2}\right)=0$ for all $i$. Applying Corollary 3.2 to $E_{1}$ along the path $L_{k-1}: z_{2} \ldots z_{k}$, and once again Lemma 3.1 for $v=z_{1}$, we obtain that, for all $i, \tilde{H}_{i}\left(E_{1}\right)=\tilde{H}_{i-\frac{2(k-1)}{3}-3}\left(\varepsilon\left(L_{m-3} \cup C_{n}\right)\right)$, which by Theorem C, Theorem B and $(*)$, is non zero only in $i=\frac{2|V|+1}{3}-2$. The Mayer- Vietoris sequence shows that $\tilde{H}_{i}(\varepsilon(G)) \neq 0$ in $i=\frac{2|V|+1}{3}-2$. Thus, in view of $(*)$, we have that $p d I(G) \geq \frac{2|V|+1}{3}$. By Lemma 2.1 the sequence $B: x_{1} z_{1}, z_{1} z_{2}+q_{0}, q_{1}, \ldots, q_{\frac{2 m}{3}-1}, z_{3} z_{4}, z_{2} z_{3}+z_{4} z_{5}, \ldots$, $z_{k-1} z_{k}, z_{k-2} z_{k-1}+z_{k} y_{1}, q_{0}^{\prime}, \ldots, q_{\frac{2 n}{3}-1}^{\prime}$, generates $I(G)$, up to radical, where the sequence $A_{m}: q_{0}, \ldots, q_{\frac{2 m}{3}-1}$ generates $I\left(C_{m}\right)$, up to radical, and the sequence $A_{n}: q_{0}^{\prime}, \ldots, q_{\frac{2 n}{3}-1}^{\prime}$ generates $I\left(C_{n}\right)$, up to radical, by Proposition 2.2. This implies that $p d I(G)=\operatorname{ara} I(G)=\frac{2|V|+1}{3}$ in this case. This completes the proof.

From Theorem 3.3 and Theorem 3.4 we deduce the following corollary.

Corollary 3.5. Let $G$ be a bicyclic graph, then ara $I\left(C_{n}\right)=p d I\left(C_{n}\right)$.

\section{REFERENCES}

1. M. Barile, On the arithmetical rank of certain monomial ideals. Preprint (2006). math.AC/0611790 Submitted.

2. M. Barile, On the arithmetical rank of the edge ideals of forests. Preprint (2006). math.AC/0607306 To appear in: Comm. Algebra.

3. M. Barile, A note on the edge ideals of Ferrers graphs. Preprint (2006). math.AC/0606353. Submitted.

4. M. Barile, On ideals generated by monomials and one binomial. Algebra Colloq. 14 (2007), 631-638.

5. M. P. Brodmann and R. Y. Sharp, Local Cohomology: an algebraic introduction with geometric applications. Cambridge University Press, Cambridge, 1998.

6. A. Corso and U. Nagel, Monomial and toric ideals associated to Ferrers graphs.

7. H. T. Ha and A. Van. Tuyl, Splittable ideals and the resolutions of monomial ideals. J. Algebra. 309 (2007), 405-425.

8. R. Hartshorne, Cohomological dimension of algebraic varieties. Ann. Math. 88 (1968), 403450 .

9. Hochster, Cohen-Macaulay rings, combinatorics, and simplicial complexes. In: Ring Theory II (B. R. McDonald and R. Morris, eds.), Lect. Notes in Pure and Appl. Math., No.26, Dekker, New York, (1977), 171-223.

10. C. Huneke and A. Taylor, Lectures on local cohomology. In: L.L. Avramov (ed.) et al., Interactions between homotopy theory and algebra. Summer school, University of Chicago, IL, USA, July 26-August 6, (2004). Contemporary Mathematics 436, American Mathematical Society, Providence, RI, 5199, (2007).

11. S. Jacques, Betti numbers of graph ideals. Ph.D. Thesis, University of Sheffield, (2004). math.AC/0410107

12. S. Jacques and M. Katzman, The Betti numbers of forests, Preprint. (2005)

13. M. Katzman, Characteristic-independence of Betti numbers of graph ideals. J. Combin. Theory Ser. A. 113 (2006), 435-454.

14. G. Lyubeznik, On the arithmetical rank of monomial ideals. J. Algebra 112 (1998), 86-89.

15. G. Lyubeznik, On the local cohomology modules $H_{A}^{i}(R)$ for ideals A generated by monomials in an R-sequence. In: Complete Intersections, Lectures given at the $1^{\text {st }} 1983$ Session of the Centro Internazionale Matematico Estivo (C.I.M.E), Acireale, Italy, June 13-21, 1983; Greco, S., Strano, R., Eds.; Springer: Berlin Heidelberg, (1984).

16. Th. Schmitt and W. Vogel, Note on set-theoretic intersections of subvarieties of projective space. Math. Ann. 245 (1979), 247-253. 
17. A. Simis, W. Vasconcelos, R. Villarreal, On the ideal theory of graphs. J. Algebra, 167, (1994), 389-416.

18. R. H. Villarreal, Monomial Algebras, Dekker. New York, NY, (2001).

Margherita Barile, Dipartimento di Matematica, Università di Bari "Aldo Moro", Via E. Orabona 4, 70125 Bari, Italy.

E-mail address: barile@dm.uniba.it

Dariush Kiani, Department of Mathematics, Amirkabir University of technology, Tehran, Iran, and School of Mathematics, Institute for Research in Fundamental SciENCES (IPM).

E-mail address: dkiani@aut.ac.ir

Fatemeh Mohammadi, Department of Mathematics, Amirkabir University of technology, Tehran, Iran.

E-mail address: f-mohammadi@cic.aut.ac.ir

Siamak Yassemi, Department of Mathematics, University of Tehran, Tehran, Iran and School of Mathematics, Institute for Research in Fundamental Sciences (IPM).

E-mail address: yassemi@ipm.ir 